Non-axisymmetric rotating-disk flows: nonlinear travelling-wave states

Hewitt, R.E. and Duck, P.W.

2000

MIMS EPrint: 2013.65

Manchester Institute for Mathematical Sciences

School of Mathematics

The University of Manchester

\footnotetext{
Reports available from: http://eprints.maths.manchester.ac.uk/

And by contacting: The MIMS Secretary

School of Mathematics

The University of Manchester

Manchester, M13 9PL, UK
} 


\title{
Non-axisymmetric rotating-disk flows: nonlinear travelling-wave states
}

\author{
By R. E. HEWITT ANd P. W. DUCK \\ Department of Mathematics, University of Manchester, Oxford Road, \\ Manchester, M13 9PL, UK
}

(Received 26 May 1999 and in revised form 15 December 1999)

\begin{abstract}
We consider the classical problem of the laminar flow of an incompressible rotating fluid above a rotating, impermeable, infinite disk. There is a well-known class of solutions to this configuration in the form of an exact axisymmetric solution to the Navier-Stokes equations. However, the radial self-similarity that leads to the 'rotatingdisk equations' can also be used to obtain solutions that are non-axisymmetric in nature, although (in general) this requires a boundary-layer approximation. In this manner, we locate several new solution branches, which are non-axisymmetric travelling-wave states that satisfy axisymmetric boundary conditions at infinity and at the disk. These states are shown to appear as symmetry-breaking bifurcations of the well-known axisymmetric solution branches of the rotating-disk equations. Numerical results are presented, which suggest that an infinity of such travelling states exist in some parameter regimes. The numerical results are also presented in a manner that allows their application to the analogous flow in a conical geometry.

Two of the many states described are of particular interest. The first is an exact, nonlinear, non-axisymmetric, stationary state for a rotating disk in a counter-rotating fluid; this solution was first presented by Hewitt, Duck \& Foster (1999) and here we provide further details. The second state corresponds to a new boundary-layer-type approximation to the Navier-Stokes equations in the form of azimuthally propagating waves in a rotating fluid above a stationary disk. This second state is a new nonaxisymmetric alternative to the classical axisymmetric Bödewadt solution.
\end{abstract}

\section{Introduction and formulation}

The analysis of flows driven by the action of a rotating boundary has long been an area of fundamental interest to a broad range of investigators. The canonical problem is the flow above a rotating disk in a rotating fluid, which has applications in areas as diverse as geophysics, crystal growth technology, rotating machinery and disk-based magnetic data storage methods.

The work of Kármán (1921) considered the incompressible flow over an infinite rotating disk in a fluid that is stationary far from the disk. It was shown that by assuming an axisymmetric radial similarity for the flow, the Navier-Stokes equations could be reduced exactly to a set of nonlinear ordinary differential equations. Numerical solution of these equations shows that the rotating disk acts to transport fluid radially outward in a boundary-layer-scale region above the disk (although there is no boundary-layer approximation necessary). The disk behaves as a centrifugal fan, with fluid being drawn vertically towards the 'fan' as fluid is transported radially outwards. 
This mechanism is crucial to the description of spin-up readjustment discussed some time later by Greenspan \& Howard (1963).

The similarity solution of von Kármán does not rely on the fluid being stationary far from the disk and the same form can be applied to the case of a rotating fluid over a stationary disk, as considered by Bödewadt (1940). In this case, the balance of centrifugal effects and the radial pressure gradient that is required for rigid body rotation is broken near to the disk resulting in a radial flow directed towards the axis of rotation and a vertical transport of fluid away from the disk.

The configurations of von Kármán and Bödewadt are two limiting cases of a more general problem, namely the axisymmetric flow of a rotating fluid over a rotating disk. When the far-field fluid and the disk are both allowed to rotate, a family of solutions can be located that has an associated parameter, $\Omega_{\infty} / \Omega_{\text {disk }}$, which is the ratio of the angular frequencies associated with the far-field fluid and the disk. The details of this more general problem were presented by Batchelor (1951), together with the related problem of the flow driven by the rotation of two parallel disks that are allowed to rotate independently. It should be noted that in the second configuration, a further parameter must be introduced, which is a Reynolds number based on the gap width of the rotating disks.

In this work we restrict attention to the more fundamental problem of the flow induced by a single infinite disk, although both the far-field fluid and the disk are allowed to rotate. The associated parameter is denoted by $\hat{W}_{e}=\Omega_{\infty} / \Omega_{\text {disk }}$, which is the ratio of the two characteristic frequencies, as described above. The flow above the disk is defined in terms of a cylindrical polar coordinate system $(r, \phi, z)$, with a radial coordinate measured from the axis of rotation, $\phi$ an azimuthal coordinate and $z$ measured parallel to the axis of rotation. The three velocity components at the point $(r, \phi, z)$ are denoted by $U, W$ and $V$, corresponding to the velocities in the radial, azimuthal and normal directions respectively.

Here we wish to investigate non-axisymmetric solutions to the classical rotatingdisk problem (and closely related flows). In this regard, it is possible to search for a solution to the Navier-Stokes equations with a radial dependence of the form first presented by von Kármán, while also allowing a dependence on the azimuthal coordinate $\phi$ :

$$
\begin{gathered}
(U, W, V)^{T}=\left(r \hat{U}(\eta, \phi, t), r \hat{W}(\eta, \phi, t), E^{1 / 2} \hat{V}(\eta, \phi, t)\right)^{T}, \\
P=r^{2} \hat{W}_{e}^{2} / 2+E Q(\eta, \phi, t) .
\end{gathered}
$$

Here $P$ is a pressure distribution, $\eta=E^{-1 / 2} z$ is a scaled coordinate, and $E=$ $v /\left(\Omega_{\text {disk }} h^{2}\right)$ is the Ekman number based on the kinematic viscosity $v$ and a natural lengthscale $h$.

For a solution in this form, the unsteady governing equations for the flow over a rotating disk are reduced to

$$
\begin{aligned}
& \hat{U}_{t}+\hat{U}^{2}+\hat{V} \hat{U}_{\eta}-\hat{W}^{2}+\hat{W} \hat{U}_{\phi}=\hat{U}_{\eta \eta}-\hat{W}_{e}^{2}+E\left\{\frac{\hat{U}_{\phi \phi}-2 \hat{W}_{\phi}}{r^{2}}\right\}, \\
& \hat{W}_{t}+2 \hat{U} \hat{W}+\hat{V} \hat{W}_{\eta}+\hat{W} \hat{W}_{\phi}=\hat{W}_{\eta \eta}+E\left\{\frac{\hat{W}_{\phi \phi}+2 \hat{U}_{\phi}-Q_{\phi}}{r^{2}}\right\},
\end{aligned}
$$




$$
\begin{gathered}
\hat{V}_{t}+\hat{V} \hat{V}_{\eta}+\hat{W} \hat{V}_{\phi}=-Q_{\eta}+\hat{V}_{\eta \eta}+E\left\{\frac{\hat{V}_{\phi \phi}}{r^{2}}\right\}, \\
2 \hat{U}+\hat{V}_{\eta}+\hat{W}_{\phi}=0 .
\end{gathered}
$$

The boundary conditions are formed from no-slip and impermeability constraints at the disk

$$
\hat{U}=\hat{V}=0, \quad \hat{W}=1 \quad \text { on } \quad \eta=0,
$$

together with a rigid-body rotation far from the disk

$$
\hat{U} \rightarrow 0, \quad \hat{W} \rightarrow \hat{W}_{e} \quad \text { as } \quad \eta \rightarrow \infty
$$

In this system, if we set $\partial / \partial \phi=0$ we obtain an exact reduction of the NavierStokes equations; the classical nonlinear, axisymmetric, rotating-disk equations are thus obtained in the form

$$
\begin{gathered}
\hat{U}^{2}+\hat{V} \hat{U}_{\eta}-\hat{W}^{2}=\hat{U}_{\eta \eta}-\hat{W}_{e}^{2}, \\
2 \hat{U} \hat{W}+\hat{V} \hat{W}_{\eta}=\hat{W}_{\eta \eta}, \\
2 \hat{U}+\hat{V}_{\eta}=0 .
\end{gathered}
$$

Although it is common to combine this system into a pair of equations, one of third and the other of second order, we shall use the formulation given above throughout this paper.

The first term in the expression for the pressure (1.2) is that required to achieve a rigid-body rotation far from the disk, while the term proportional to the Ekman number can be determined separately.

The solution structure of (1.9)-(1.11) has been discussed by many authors. In particular, it has been shown that an infinity of solution branches can be found in the vicinity of $\hat{W}_{e}=0$, and similar non-uniqueness can be located around a singular solution structure near $\hat{W}_{e} \approx \pm 1.4355$. The exact form of the non-uniqueness and the relationship between the successive solution branches will not be dealt with in detail here; the reader is referred to the review article of Zandbergen \& Dijkstra (1987) for further details.

A convenient measure of the solutions to (1.9)-(1.11) is the value of $\hat{V}$ at the edge of the 'boundary layer' $\uparrow$. We shall refer to this quantity as $\hat{V}_{\infty}$, which is formally defined to be the limiting value of $\hat{V}(\eta)$ as $\eta \rightarrow \infty$. In terms of 'spin-up' problems this quantity can be interpreted as the 'Ekman suction', that is, the flow into or out of the layer forced by a net radial inflow/outflow near to the disk surface. It is readily seen from the form of (1.9)-(1.11) that the governing equations remain unchanged under the transformations $(\eta \rightarrow-\eta, \hat{V} \rightarrow-\hat{V})$, due to a reflectional symmetry about the plane of the disk. In this paper we consistently assume $\eta \in[0, \infty)$, in which case, if $\hat{V}_{\infty}$ is negative the flow is directed towards the disk surface.

The first four solution branches to the equations (1.9)-(1.11) in the neighbourhood of $\hat{W}_{e}=0$ are shown in figure $1(a)$; these solutions can be extended to an infinity of such solutions past the point (ii) noted in the figure. It is sufficient here to note that these higher solution branches require successively larger domains and hence further care is required in the numerical method. The solution branch may also be continued beyond the point (i) shown in the figure, through $\hat{V}_{\infty}=0$ at $\hat{W}_{e}=1$ (the trivial

$\dagger$ Although there is no formal boundary-layer approximation required at this stage, we shall still refer to the region as a boundary layer for convenience. 

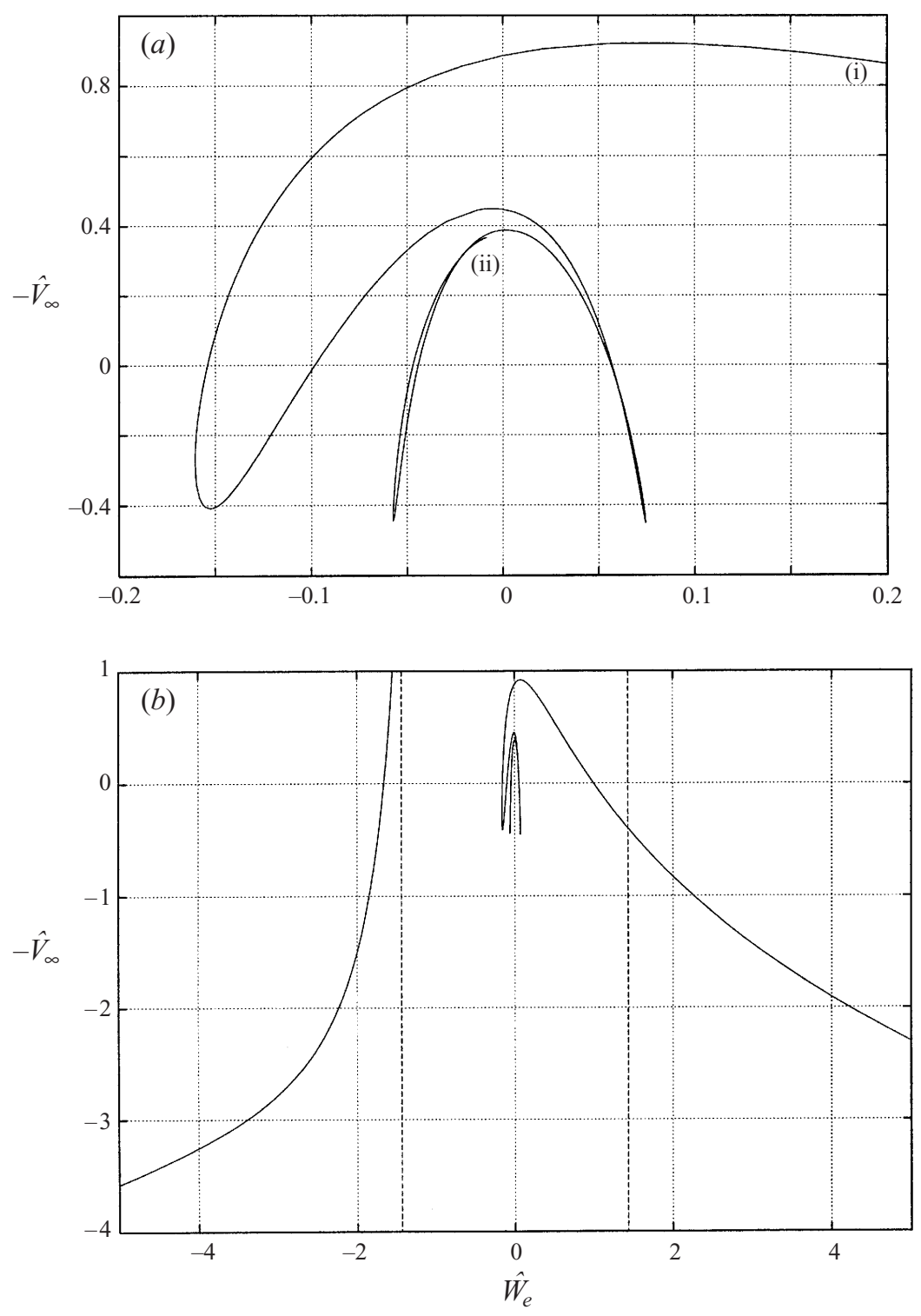

FIGURE 1. The solution branches of the axisymmetric rotating-disk equations. (a) The first four solution branches (near $\hat{W}_{e}=0$ ). (b) The branches of $(a)$ are shown near to $\hat{W}_{e}=0$ together with the singular solution branch near $\hat{W}_{e} \approx-1.4335$ (as marked by the vertical dashed line). A further collection of isolated solution branches can be located near to $\hat{W}_{e} \approx \pm 1.4335$, but are not shown here.

solution of rigid-body rotation) to the limiting Bödewadt solution as $\hat{W}_{e} \rightarrow \infty$. The behaviour over a larger range of $\hat{W}_{e}$ is displayed in figure $1(b)$, which shows the same solution branches over a broader range of $\hat{W}_{e}$, together with a further solution that becomes singular at $\hat{W}_{e} \approx-1.4355$. This solution can be continued from the singular solution structure, as shown, to larger negative values of $\hat{W}_{e}$, again connecting to the Bödewadt solution as $\hat{W}_{e} \rightarrow-\infty$. A similar isolated singular solution can be found at $\hat{W}_{e} \approx+1.4355$, and higher-branch solutions can be found in the neighbourhood 
of \pm 1.4355 that we do not show here; the reader is again referred to the paper of Zandbergen \& Dijkstra (1987) for further details.

The above details concerning classical axisymmetric rotating-disk flows are well known and presented here for later reference. This work is concerned with the extension of the previously detailed solution branches to include the possibility of non-axisymmetric flow states that still satisfy axisymmetric boundary conditions. In this manner, we wish to look for a broader class of solution to the same physical problem by relaxing our assumption concerning the symmetry of the flow. We note here that Lai, Rajagopal \& Szeri (1985) have made reference to non-axisymmetric rotating-disk flows; however their solutions, though valid, are somewhat less natural than those discussed herein. Only one class of flow discussed by Lai et al. is open to physical interpretation and corresponds to a rotating disk in a fluid with a uniform translation far from the disk.

The solutions we present here are more simply interpreted, and are closely related to a weakly nonlinear temporal stability analysis of the classical rotating-disk states to azimuthally propagating waves of the same self-similar form. In this sense, the analysis presented herein is connected to the work of Hall, Balakumar \& Papageorgiou (1992), which effectively considered the stability of von Kármán's solution $\left(\hat{W}_{e}=0\right.$ in our notation) to finite-amplitude, unsteady disturbances with an azimuthal wavenumber of two.

The recent work of Hewitt, Duck \& Foster (1999, hereafter referred to as HDF) considered the steady, axisymmetric boundary-layer solutions for a swirling stratified fluid in a rotating cone. This work made use of a similarity-type solution of von Kármán form, and the governing equations were shown to reduce to the axisymmetric rotating-disk equations in a sub-region of parameter space (as buoyancy forces were removed and the cone flattened into a disk). In the work of HDF, a new steady, exact solution to the Navier-Stokes equations was presented, corresponding to a non-axisymmetric flow above a rotating disk. In Appendix A we provide further brief details concerning the properties of this exact solution and, in Appendix B, show how the consideration of a more general boundary-layer flow inside a conical container (rather than above a rotating disk) motivates the consideration of azimuthally propagating waves and hence leads to the discovery of further boundary-layer solutions relevant to the flow over a rotating disk.

The format of this paper is as follows. In $\S 2$, we give details of the method used to determine the location of bifurcations to non-axisymmetric, travelling-wave states. The main interest here is in states corresponding to azimuthally propagating waves; however these results are complimentary to (and indeed motivated by) the existence of the aforementioned exact, non-axisymmetric stationary solution, as described in Appendix A. In $\S 3$, we provide some numerical details regarding the computation of fully nonlinear, non-axisymmetric states over broad ranges of the parameter $\hat{W}_{e}$. Finally, in $\S 4$, we give some conclusions and comment on the stability of the new solution branches.

\section{Bifurcations to travelling-wave states}

It must first be noted that all terms have been retained in the system (1.3)-(1.6), and non-axisymmetric solutions of this form will (in general) require a boundary-layer approximation before progress can be made. The approximation is straightforward, under the assumption that $E \ll 1$, such that the velocity field can be formally expanded to provide a leading-order system equivalent to that noted above but neglecting the 


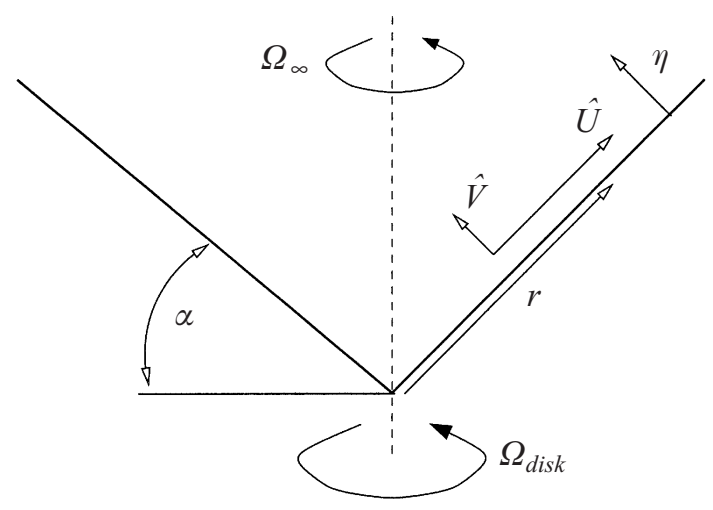

FIGURE 2. The analogous coordinate system in a conical geometry.

bracketed terms. However, there is a particular exact form of stationary solution to (1.3)-(1.6) that has been noted previously by HDF, further details of which are presented in Appendix A. As we shall show, this exact solution occurs as a special case in a class of more general (travelling-wave) states.

\subsection{Periodic, non-axisymmetric states}

The initial motivation for the work we present here comes from the observation of transient, non-axisymmetric flows in a conical geometry during spin-down experiments. If non-axisymmetric states can be located in the more fundamental problem of an un-stratified, rotating-disk flow, then the continuation to a more general conical boundary-layer with a stratified fluid is a straightforward procedure. With this in mind, we discuss in Appendix B how the governing system of equations changes if a conical geometry is considered rather than a flat disk. In general we may consider a conical container with sidewalls that are at an angle of $\alpha$ to the horizontal, with $\alpha=0$ providing the rotating-disk results (see figure 2 ).

One immediate consequence of the analysis of Appendix B is that bifurcations to a stationary solution only exist in two special cases, that is, for a flat disk and a cone with walls at an angle of $\alpha=\pi / 3$ to the horizontal. Since the geometry for this problem can be changed in a continuous manner from the flat-disk case to the conical case, it is unclear how the bifurcating, stationary, non-axisymmetric solution can be continued in the cone-angle parameter $\alpha$. In particular, when the geometry is perturbed away from that of a flat disk into a conical shape with small wall angle, the eigenrelation (B 10) is no longer satisfied and a bifurcated stationary solution cannot be located. To relocate a bifurcation to a non-axisymmetric state for a conical geometry a further free parameter is required. To resolve this apparent difficulty we are forced to consider a broader class of (boundary-layer) solution, allowing for states that are azimuthally propagating waves. This is accomplished through the introduction of an additional parameter, namely $\Omega$, which corresponds to the frequency of the wave.

As discussed in Appendix B, the corresponding boundary-layer system in a conical geometry is simply obtained from (1.3)-(1.6) by neglecting terms of $O(E)$ and making the transformation $\partial / \partial \phi \rightarrow \beta^{-1} \partial / \partial \phi$ (where $\beta=\cos \alpha$ ). A solution can then be sought in the form

$$
(\hat{U}, \hat{W}, \hat{V})^{T}=\left(U_{0}(\eta), W_{0}(\eta), V_{0}(\eta)\right)^{T}+\epsilon(\tilde{U}(\eta), \tilde{W}(\eta), \tilde{V}(\eta))^{T} \exp \{\mathrm{i}(n \phi+\Omega t)\},
$$

with $\epsilon \ll 1$. As before, at $O(\epsilon)$ there is a linear eigenvalue problem to be solved 
for the critical value of $\hat{W}_{e}$ at which a bifurcation to a travelling-wave state can be located. In this case the eigenvalue relation to be satisfied is of the form

$$
\mathscr{G}\left(\hat{W}_{e}, \lambda, \Omega\right)=0,
$$

where $\lambda$ denotes the quantity $n \beta^{-1}=n / \cos \alpha$.

The condition (2.2) determines a functional relationship between the three real quantities $\hat{W}_{e}, \lambda$ and $\Omega$. It can be approached in two distinct ways. One method is to specify a real value for $\hat{W}_{e}$ and solve the $O\left(\epsilon^{0}\right)$ rotating-disk equations, then one can determine a complex value for $\lambda$ for a given real frequency $\Omega$. If $\lambda$ can be made real for a given combination of $\hat{W}_{e}$ and $\Omega$, then at this critical value of $\hat{W}_{e}$ we may expect a bifurcation to a travelling-wave state with an integer wavenumber $n$, provided that

$$
\lambda=n \beta^{-1},
$$

for some real cone angle $\alpha$, where $\beta=\cos \alpha$.

The second approach is essentially that of a linear temporal stability analysis of the rotating-disk equations. For a specified axisymmetric solution at a fixed value of $\hat{W}_{e}$, one can determine a complex frequency $\Omega$ for a real $\lambda$. The stability of the axisymmetric solution to modes of 'wavenumber' $\lambda$ is then determined by the sign of $\Omega_{i}$ (the imaginary part of $\Omega$ ) with $\Omega_{i}<0$ indicating temporal instability.

The advantage of notionally considering a complete class of conical geometries with $\alpha \in[0, \pi / 2)$ is that one can solve the same equations relevant to the flat-disk geometry but allow the wavenumber to be a real value (rather than an integer). This can be allowed with the understanding that, for a given real $\lambda>1$, the solution is physically appropriate (i.e. satisfies the azimuthal periodicity constraint) in a sequence of conical geometries of angle $\alpha=\arccos (n / \lambda)$ with an integer wavenumber $n$.

Although a solution to (2.2) cannot be found with $n$ an integer for a general $\alpha$ with $\Omega=0$, by allowing for a non-zero $\Omega$ there is no difficulty in determining how the bifurcation point varies as $\alpha$ is continued from $\alpha=0$ (the flat-disk case). We show the relationship between $\lambda=n / \cos \alpha$ and $\hat{W}_{e}$ in figure $3(a)$, together with $\Omega$ and $\hat{W}_{e}$ in $3(b)$. The bifurcation to an exact non-axisymmetric solution described in Appendix A occurs at $\lambda=2$ (i.e. $n=2, \alpha=0$ or $n=1, \alpha=\pi / 3$ ) at which point $\hat{W}_{e} \approx-0.14485$ (shown as the dashed line) and $\Omega=0$.

On perturbing $\alpha$ away from zero, or equivalently varying $\lambda$, the bifurcation point can be seen to move to a different location on the axisymmetric rotating-disk solution branch (that is, to a different value of $\hat{W}_{e}$ ). However, the states arising from the bifurcation point at $\alpha \neq 0$ are typically travelling solutions with $\Omega \neq 0$.

The two numbered points (i, ii) noted in both $(a)$ and $(b)$ of figure 3 correspond to the same axisymmetric solution to the rotating-disk equations. This point is at $\hat{W}_{e} \approx-0.1605$ and corresponds to the limit point of the solution branches shown in figure 1.

We have already mentioned that one can view the bifurcation analysis as corresponding to a linear temporal stability analysis of the axisymmetric solutions. A stability analysis of this form has been presented before for perturbations that are axisymmetric by Bodonyi \& $\mathrm{Ng}(1984)$. It was shown that there is a stability transition (to unsteady axisymmetric perturbations) at the 'nose' of the solution branch shown in figure 1 , where $\hat{W}_{e} \approx-0.1605$. It is interesting to note that the mode that gives rise to the exact, $n=2$ non-axisymmetric stationary state at $\hat{W}_{e} \approx-0.14485$ may be regarded as a non-axisymmetric continuation of the same mode located by Bodonyi $\& \mathrm{Ng}$. As shown in figure 3 , on approaching the limit point denoted by (i), $\lambda \rightarrow 0$ and $\Omega \rightarrow 0$, reproducing the neutrally stable axisymmetric eigenmode of Bodonyi \& $\mathrm{Ng}$. 

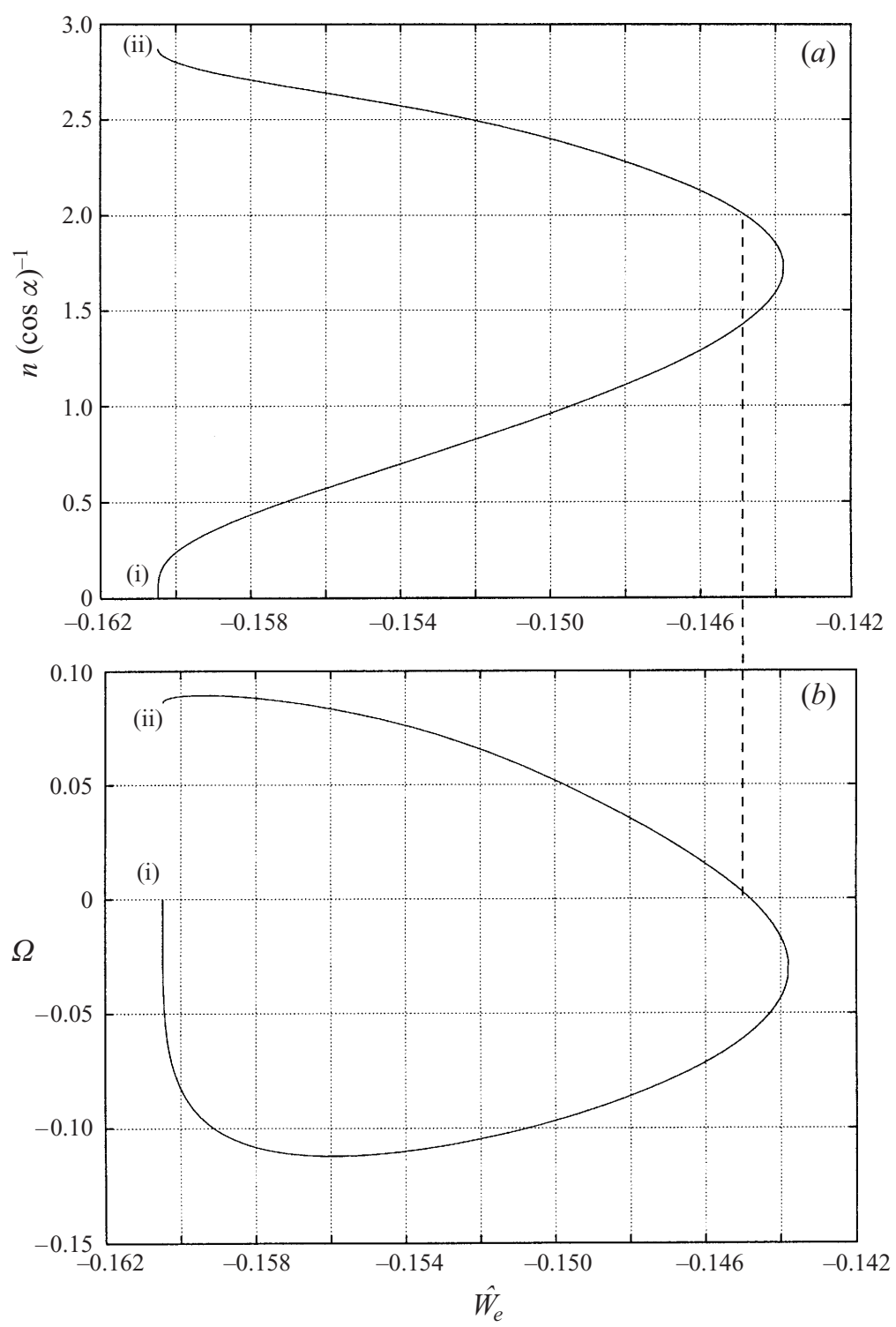

FIGURE 3. The location of the bifurcation point for varying values of $\lambda=n / \cos \alpha$ and frequency $\Omega$. When $\hat{W}_{e} \approx-0.14485$ there is a bifurcation to a $\lambda=2, \Omega=0$ (stationary) mode, as described in Appendix A.

At this same point on the axisymmetric solution branch, there is another neutrally stable mode with $\lambda \approx 2.9$ and $\Omega \approx 0.08$, denoted as point (ii) in figure 3 . This second mode is only relevant to a set of conical geometries however, since $\lambda$ is not an integer and therefore azimuthal periodicity cannot be achieved for a flat disk.

Our primary concern however is with bifurcations to new states relevant to the flow over a flat disk, i.e. $\alpha=0$, for which we must have $\lambda=n$, an integer azimuthal wavenumber. It can be easily observed from figure 3 that continuation of the $\lambda=2$ bifurcation reveals a further bifurcation to a non-axisymmetric travelling-wave state 
with a wavenumber of unity at $\hat{W}_{e} \approx-0.1493$. The bifurcated state is an azimuthally propagating wave, with a frequency of $\Omega \approx-0.09$.

It is the presence of this second $n=1$ travelling-wave solution that resolves the apparent confusion over the presence of the bifurcation to stationary states with $n=2$ when $\alpha=0$ and $n=1$ when $\alpha=\pi / 3$ (both occurring at $\hat{W}_{e} \approx-0.14485$, as described in Appendix B). When allowing for non-axisymmetric solutions that travel circumferentially, we observe that for the flat-disk case $(\alpha=0)$ there are at least two bifurcation points: one is stationary with a wavenumber $n=2$ while the other is a travelling solution with a wavenumber of $n=1$. If we follow these bifurcated states while increasing $\alpha$ from the flat-disk case to an angle of $\alpha=\pi / 3$, then we can immediately observe from figure 3 that the $n=2$ state begins to travel with an increasing frequency, while the $n=1$ state travels more slowly. When $\alpha$ is increased through $\alpha=\pi / 3$, the $(n=1)$ state that was 'previously' travelling becomes stationary before beginning to travel in the opposite sense as the cone angle is increased further.

\subsection{Bifurcations from higher-branch axisymmetric states}

As shown in figure 3, the bifurcation to a non-axisymmetric state can be continued for varying real wavenumber $\lambda$, revealing a further travelling state with $n=1$ at $\hat{W}_{e} \approx-0.1492$. The numerical results of figure 3 terminate when $\hat{W}_{e} \approx-0.1605$, that is, at the limit point of the branch-1 solution shown in figure 1 . However, there is no difficulty in continuing the calculations onto successive branches of the axisymmetric rotating-disk equations. In this manner we can obtain the results of figure 4, which shows the same data presented by figure 3 but continued further onto the second branch shown in figure 1 .

As can be seen from figure 4 , when $\lambda$ is increased further the bifurcation point moves to the second branch and yet more modes relevant to the flat-disk geometry are revealed (where $\lambda$ is an integer). In particular, there is a bifurcation to an $n=3$ state with $\Omega \approx 0.08$ at $\hat{W}_{e} \approx-0.1588$. Similarly, bifurcations to modes with an azimuthal wavenumber $n=4$ can be found on the second branch at $\hat{W}_{e} \approx \pm 0.06$.

There is an established literature (see Zandbergen \& Dijkstra 1987 for a list of references) on the structure of the higher-branch axisymmetric solutions to the rotating-disk equations. The most notable feature of these states is the relationship of a solution on the $m$ th branch to the corresponding $(m-1)$-branch solution. When states from consecutive branches are compared they are found to differ only in a region displaced from the boundary. In fact, the $m$-branch solution is formed from the $(m-1)$-branch solution with the addition of an extra 'inviscid hump' into the velocity profiles.

As a consequence of the inviscid-cell-type structure of these higher branches, if the eigenfunctions in the bifurcation analysis decay sufficiently rapidly with the boundarylayer coordinate, there will be an analogous degeneracy of the bifurcated states. In particular, if a bifurcation point can be located on the $(m-1)$ branch corresponding to some eigenmode, then the same mode is also appropriate to the $m$ th branch since the addition of a further 'inviscid hump' at the end of the velocity profiles simply acts to broaden the range of possible eigensolutions. Numerical results confirm this scenario, with the eigenfunctions rapidly approaching zero within the extent of the hump. We therefore note that, at least when considering weakly non-axisymmetric bifurcated states, an infinity of such solutions are likely to exist, again distinguished by the addition of an extra inviscid cell. However, these (axisymmetric) higher-branch states are themselves all increasingly unstable to axisymmetric perturbations and have rather aphysical velocity profiles. 

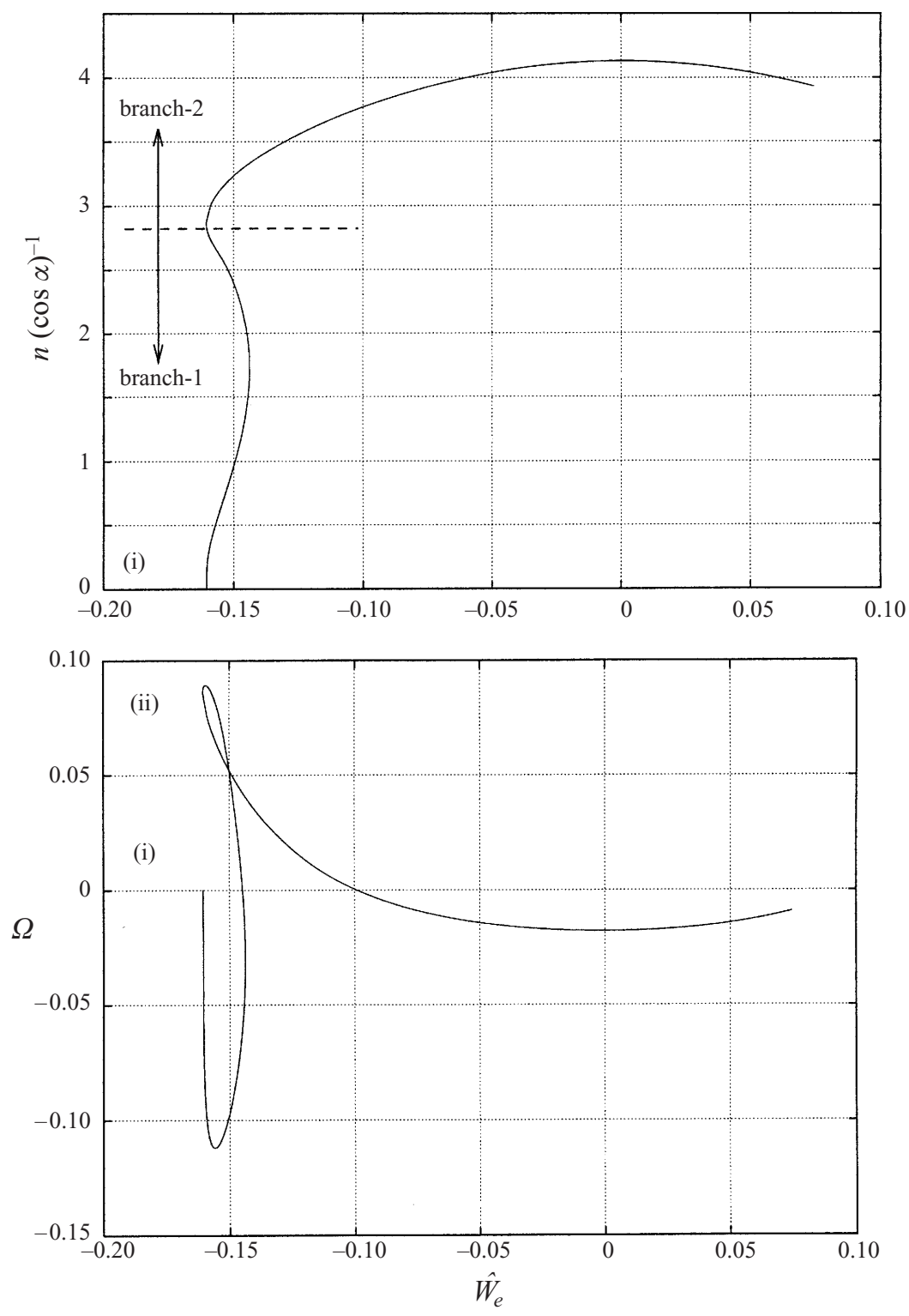

FIGURE 4. The location of the bifurcation point for varying values of $\lambda=n / \cos \alpha$ and frequency $\Omega$. The bifurcation point here has been continued onto the second branch of solution shown in figure 1.

So far in this work we have presented details of just the first five bifurcations found near the 'nose' of the solution branches of figure 1 . However, the computations can be extended to locate further bifurcations to non-axisymmetric states of higher azimuthal wavenumber by applying the same techniques discussed previously; these other states arise from the higher-branch axisymmetric solutions. In general, one can determine a set of complex eigenvalues $\{\Omega\}$ for given integer values of $\lambda$ at any point on the axisymmetric solution branches. Bifurcation points can then be located via a local search technique, varying $\hat{W}_{e}$ along the higher-branch states until $\Omega_{i}=0$. 


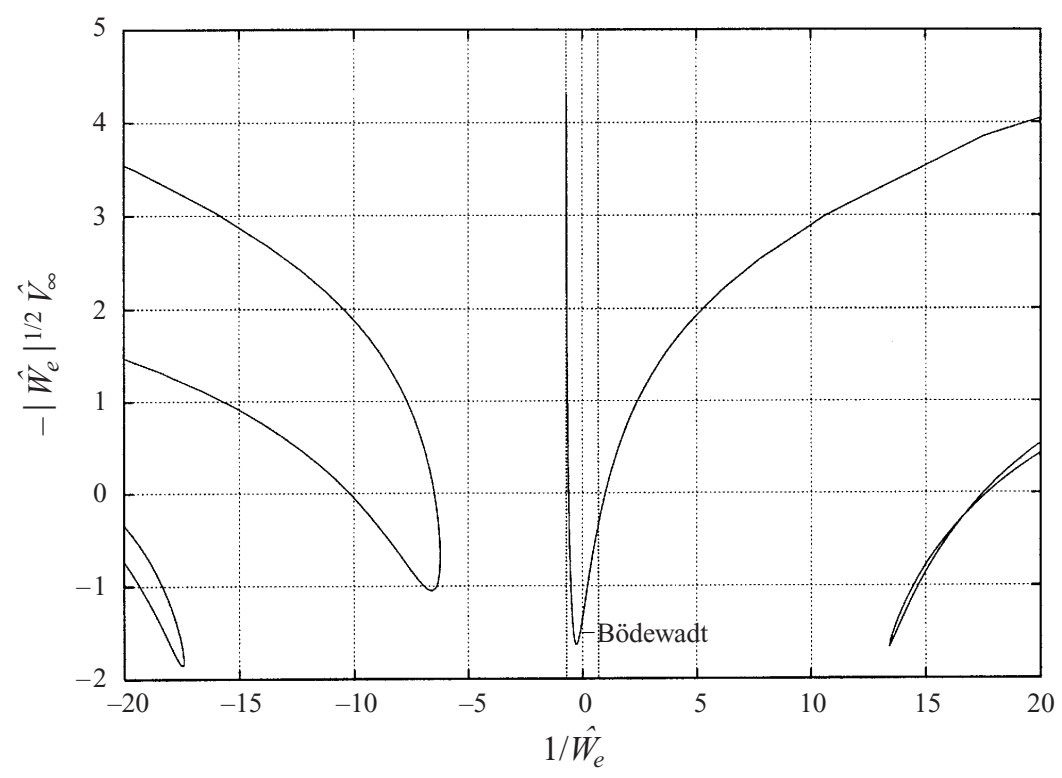

FIGURE 5. The axisymmetric solution branches in the limit of $\hat{W}_{e} \rightarrow \infty$. The Bödewadt solution is the state shown at $1 / \hat{W}_{e}=0$. The pair of vertical lines is at $1 / \hat{W}_{e}= \pm(1.4335)^{-1}$, which are values near which a singular structure can be found.

We do not present any details regarding these additional states beyond noting their existence.

\subsection{Bifurcations near the Bödewadt limit}

Thus far we have concentrated on seeking bifurcating non-axisymmetric solutions in the $\hat{W}_{e} \approx 0$ region of parameter space. However, as shown in figure $1(b)$, at least one axisymmetric solution can be located at all values of $\hat{W}_{e}$ outside the interval $(-1.4335,-0.1605)$.

The von Kármán solution is found at $\hat{W}_{e}=0$, but there is an equally important limiting solution as $\hat{W}_{e} \rightarrow \pm \infty$ : the Bödewadt solution. It is easy to observe from the rotating-disk equations (1.9)-(1.11), that as $\left|\hat{W}_{e}\right| \rightarrow \infty$, one can rescale in the following manner:

$$
\begin{gathered}
\hat{U}=\hat{W}_{e} U^{*}, \\
\hat{W}=\hat{W}_{e} W^{*}, \\
\hat{V}=\left|\hat{W}_{e}\right|^{1 / 2} V^{*}, \\
\frac{\partial}{\partial \eta}=\left|\hat{W}_{e}\right|^{1 / 2} \frac{\partial}{\partial \eta^{*}} .
\end{gathered}
$$

This leads to a system equivalent to (1.9)-(1.11), but parameterized by $1 / \hat{W}_{e}=$ $\Omega_{\text {disk }} / \Omega_{\infty}$. In this rescaled system, the boundary conditions remain the same except $W^{*}=1 / \hat{W}_{e}$ on $\eta^{*}=0$ and $W^{*} \rightarrow 1$ as $\eta^{*} \rightarrow \infty$.

Figure 5 shows the same solution branches presented in figure $1(b)$, but in this different parameterization. In this case, the von Kármán solution is obtained by taking $1 / \hat{W}_{e} \rightarrow \pm \infty$ and the Bödewadt solution is at $1 / \hat{W}_{e}=0$, corresponding to a rotating fluid over a stationary disk. Note that for the Bödewadt solution, the axial 
flow (at large values of $\eta^{*}$ ) is directed away from the disk since the net mass transport in the 'boundary-layer' is radially inwards.

Given the above formulation, one can again seek bifurcations to non-axisymmetric travelling-wave states by solving an analogous eigenvalue problem to (2.2). In this manner we have located a bifurcation to a new state with an azimuthal wavenumber $n=1$, and frequency $\Omega \approx 0.87$ at $1 / \hat{W}_{e} \approx 0.06$. Continuation of the non-axisymmetric state arising from this bifurcation will be shown in the following section to lead to a new nonlinear boundary-layer solution to the Bödewadt configuration $\left(1 / \hat{W}_{e}=0\right)$.

\section{Fully nonlinear travelling-wave states: numerical results}

Having located a number of bifurcation points on the axisymmetric rotating-disk solution branches, we now compute the nonlinear travelling-wave states that arise from these critical points. Details of the nonlinear, exact, stationary state are shown in Appendix A; however, the more general travelling-wave boundary-layer solutions are much more complex to determine.

Starting with the non-axisymmetric rotating-disk equations, we employ a boundarylayer approximation, assuming that $E \ll 1$. A solution of (1.3)-(1.6) with $E=0$ is then sought in the form

$$
\hat{\boldsymbol{U}}=\sum_{m=-\infty}^{\infty} \hat{\boldsymbol{U}}_{m} \exp \{\operatorname{im}(\lambda \phi+\Omega t+\Delta)\}
$$

where $\Delta$ is an arbitrary phase and

$$
\hat{\boldsymbol{U}}=(\hat{U}, \hat{W}, \hat{V})^{T}, \quad \hat{\boldsymbol{U}}_{m}=\left(\hat{U}_{m}, \hat{W}_{m}, \hat{V}_{m}\right)^{T} .
$$

In this expression, we truncate the series at $m= \pm N$ (the maximum number of Fourier modes), $\hat{\boldsymbol{U}}_{m}$ are complex, $\lambda$ is a real 'wavenumber' and $\Omega$ is a real frequency. Here, the wavenumber $\lambda$ is as discussed in $\S 2$, and can be allowed to be real (rather than an integer) when considering a conical geometry. For this discussion however, we shall only consider the nonlinear branches relevant to a flat disk, for which $\lambda$ must be an integer.

The decomposition (3.1) results in a $5 \times(2 N+1)$-th order system

$$
\begin{gathered}
\mathrm{i} \Omega U_{n}-U_{n}^{\prime \prime}+\hat{W}_{e}^{2} \delta_{0 n}+\sum_{m=-N}^{N} U_{n-m} U_{m}+V_{n-m} U_{m}^{\prime}-W_{n-m} W_{m}+\mathrm{i} m \lambda W_{n-m} U_{m}=0, \\
\mathrm{i} \Omega W_{n}-W_{n}^{\prime \prime}+\sum_{m=-N}^{N} 2 U_{n-m} W_{m}+V_{n-m} W_{m}^{\prime}+\mathrm{i} m \lambda W_{n-m} W_{m}=0, \\
2 U_{n}+V_{n}^{\prime}+\mathrm{i} n \lambda W_{n}=0,
\end{gathered}
$$

where $\delta_{0 n}=1$ if $n=0$ and is zero otherwise, and a similar expression can be obtained for the pressure correction $Q(\eta, \phi, t)$. The boundary conditions are

$$
\hat{U}_{0, \ldots, N}=\hat{V}_{0, \ldots, N}=0, \quad \hat{W}_{0}=1 \quad\left(\text { or } 1 / \hat{W}_{e}\right), \quad \hat{W}_{1, \ldots, N}=0 \quad \text { on } \quad \eta=0,
$$

and

$$
\hat{U}_{0, \ldots, N} \rightarrow 0, \quad \hat{W}_{0} \rightarrow \hat{W}_{e} \quad(\text { or } 1), \quad \hat{W}_{1, \ldots, N} \rightarrow 0 \quad \text { as } \quad \eta \rightarrow \infty .
$$

Here, the alternative boundary conditions on $\hat{W}$ are those appropriate to the rescaled Bödewadt limit, for which the $\hat{W}_{e}^{2}$-term in (3.3) must be replaced by unity. 
This system is solved by Newton iteration following central differencing in $\eta$. To force a non-axisymmetric solution we specify an amplitude measure for the first harmonic in the form

$$
\hat{U}_{1}^{\prime}(0)=A,
$$

where $A$ can be made real without any loss of generality, corresponding to choosing the phase $\Delta$ in (3.1). Since this extra condition has been imposed on the system, we temporarily neglect the no-slip condition for the first harmonic.

Two methods of solving the difference equations were utilized. One method fixes any two of the four real quantities $\left\{\hat{W}_{e}, A, \lambda, \Omega\right\}$ and iterates on the remaining two in order to satisfy the previously neglected (complex) no-slip condition $\hat{U}_{1}(0)=0$. A second approach allows $\Omega=\Omega_{r}+\mathrm{i} \Omega_{i}$ to be a complex variable, which can be iterated upon to satisfy the complex no-slip condition for any given $A, \hat{W}_{e}, \lambda$ (all real). A nonlinear equilibrium state is then located in this latter approach by iterating on one of the real variables $\left\{A, \hat{W}_{e}, \lambda\right\}$ to force $\Omega_{i}=0$. On specifying $\hat{W}_{e}$ and $\lambda$, with $A \ll 1$, this second method corresponds to a linear stability analysis of the axisymmetric solutions to non-axisymmetric modes of wavenumber $\lambda$.

We now describe some of the nonlinear states that arise from the previously described bifurcation points in the case $\alpha=0$ (a flat-disk geometry).

$$
\text { 3.1. The } n=1 \text { state, near } \hat{W}_{e} \approx-0.1492
$$

This $n=1$ solution can be found in the neighbourhood of $\hat{W}_{e} \approx-0.1492$ for the first branch of the axisymmetric solutions. By utilizing the numerical procedures detailed above we have continued a non-axisymmetric travelling-wave solution away from this critical value of $\hat{W}_{e}$.

In figure 6(a) we show the mean component of the axial velocity far from the disk, $\hat{V}_{0}(\eta \rightarrow \infty)$, for this new branch of solution over a range of $\hat{W}_{e}$. Figure $6(b)$ shows the variation in $A=\hat{U}_{1}^{\prime}(0)$, of the travelling-wave state as the branch is continued to larger amplitudes. It is a trivial exercise to show that near to the bifurcation point we have the scalings

$$
A \sim\left(\hat{W}_{e}-\hat{W}_{b i f}\right)^{1 / 2}, \quad \Omega \sim \Omega_{b i f}+O\left(\hat{W}_{e}-\hat{W}_{b i f}\right),
$$

where $\hat{W}_{e}=\hat{W}_{b i f}$ is the bifurcation point, at which the frequency of the wave solution is $\Omega_{b i f}$.

In figure 7, we show the behaviour of two different measures of the individual modes as $\hat{W}_{e}$ is varied along the nonlinear non-axisymmetric branch. The first measure, figure $7(a)$, is the axial velocity component far from the disk surface,

$$
\hat{V}_{n \infty}=\hat{V}_{n}(\eta \rightarrow \infty)+\hat{V}_{-n}(\eta \rightarrow \infty) .
$$

The second measure, as shown in figure $7(b)$, is an integral quantity,

$$
E_{n}=\frac{1}{2} \int_{\eta=0}^{\infty}\left(\hat{U}_{n}+\hat{U}_{-n}\right)^{2}+\left(\hat{W}_{n}+\hat{W}_{-n}\right)^{2} \mathrm{~d} \eta .
$$

In these figures, although the 'amplitudes' of the higher modes are increasing, there is no obvious evidence of any difficulty associated with the series (3.1). However, as can be observed from figure 6(a), the solution branch appears to terminate at a finite value of $\hat{W}_{e}$. We have been unable to continue this $n=1$ state to any further values of $\hat{W}_{e}$ and it is our conjecture that the series (3.1) does in fact fail on approaching some critical value of $\hat{W}_{e}$. We note that the solution could not be continued despite a large 

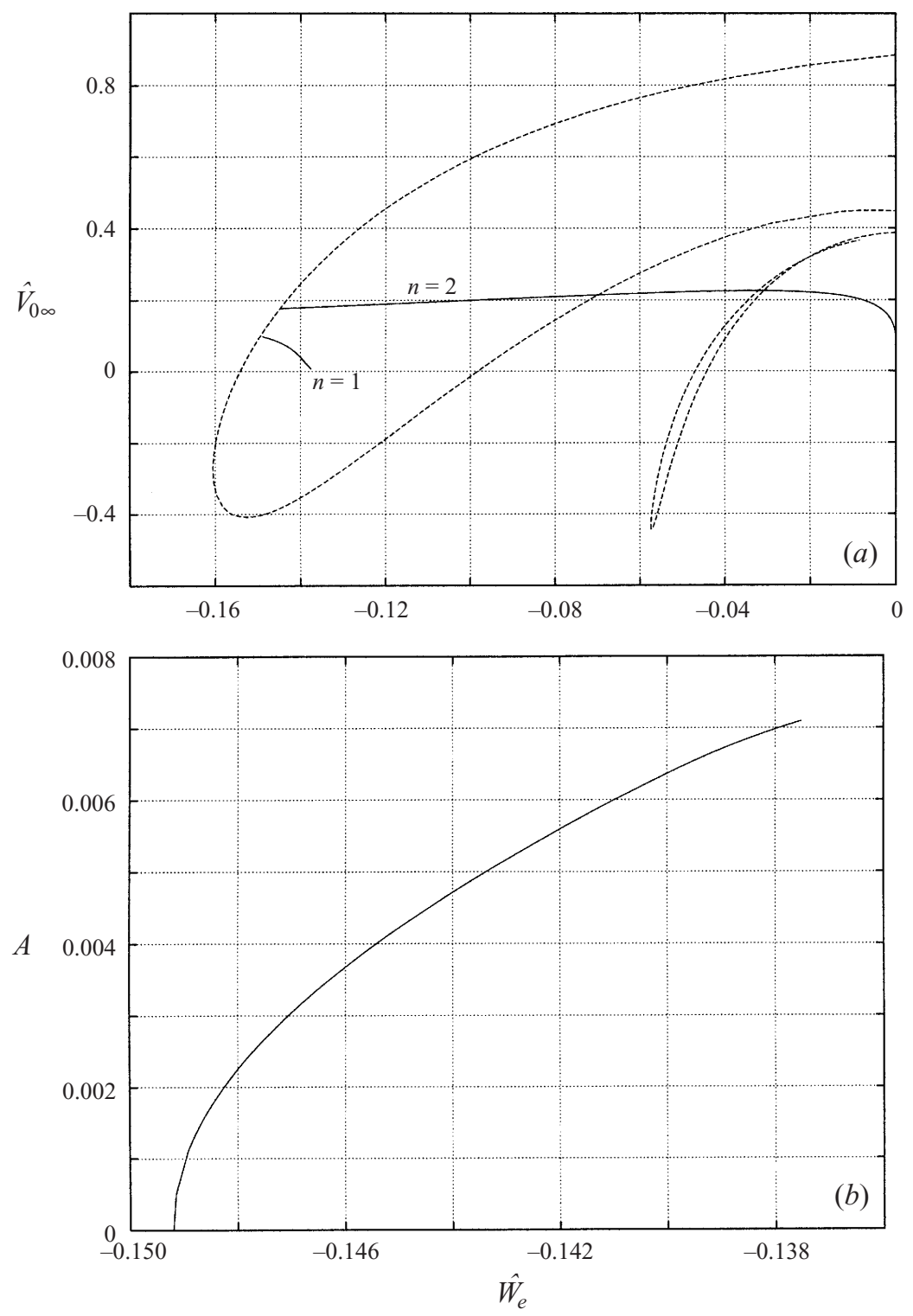

FIGURE 6 . The $n=1$ nonlinear travelling-wave solution for a flat-disk geometry: $(a)$ the 'location' of the nonlinear branch, measured by the mean axial flow far from the disk surface; $(b)$ the amplitude $A=\hat{U}_{1}^{\prime}(0)$ of the nonlinear state.

number of computations, covering a broad range of domain sizes, grid sizes, number of modes and a reduction of the steps in $\hat{W}_{e}$ during the continuation procedure.

To provide some numerical evidence towards our conjecture that the decomposition (3.1) fails, we present some further results in figure 8. Figure 8(a) again shows the quantities $\hat{V}_{n \infty}$, but scaled by their weakly nonlinear descriptions. If the nonaxisymmetric state is in some sense weakly nonlinear, then one might expect that an amplitude measure of the $n$th mode would behave in the following manner:

$$
\hat{V}_{n \infty} \sim c_{n}\left(\hat{W}_{e}-\hat{W}_{b i f}\right)^{n / 2},
$$



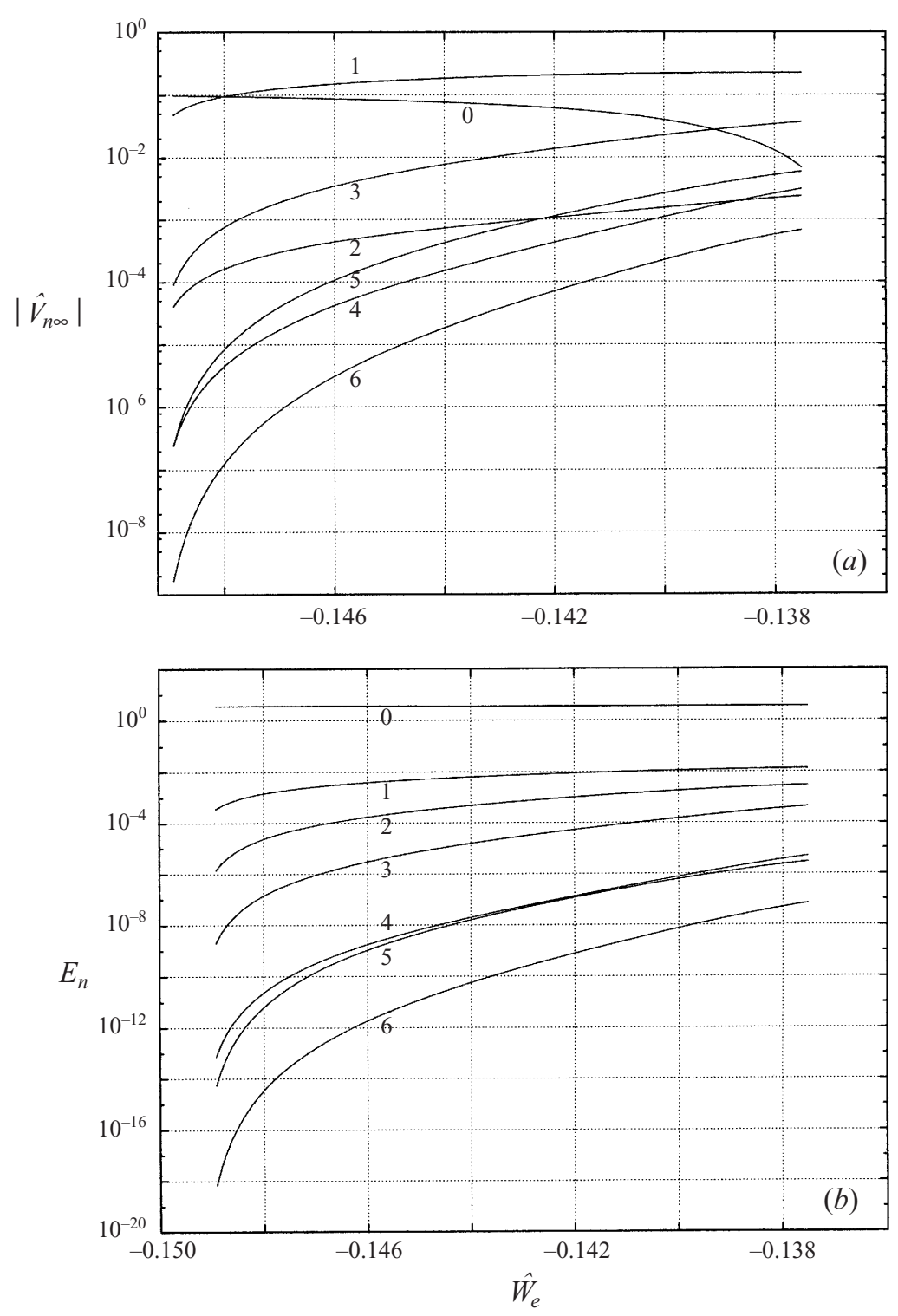

FIGURE 7. The $n=1$ nonlinear travelling-wave solution for a flat-disk geometry. (a) The axial flow at infinity for the $n$th mode. (b) An integral measure of the $n$th mode. The series (3.1) was truncated at $N=6$ in these calculations.

where $\left\{c_{n}\right\}$ is a set of real coefficients. If the non-axisymmetric solution branch remains only weakly nonlinear, then the ratio

$$
\frac{c_{1}^{n} \hat{V}_{n \infty}}{c_{n} \hat{V}_{1 \infty}^{n}}
$$

should remain nearly order one. A similar argument can be applied to any amplitude measure, and in particular to the quantities $E_{n}$, which are shown in figure $8(b)$.

As can be observed from figure 8 , the higher modes are increasingly sensitive to 

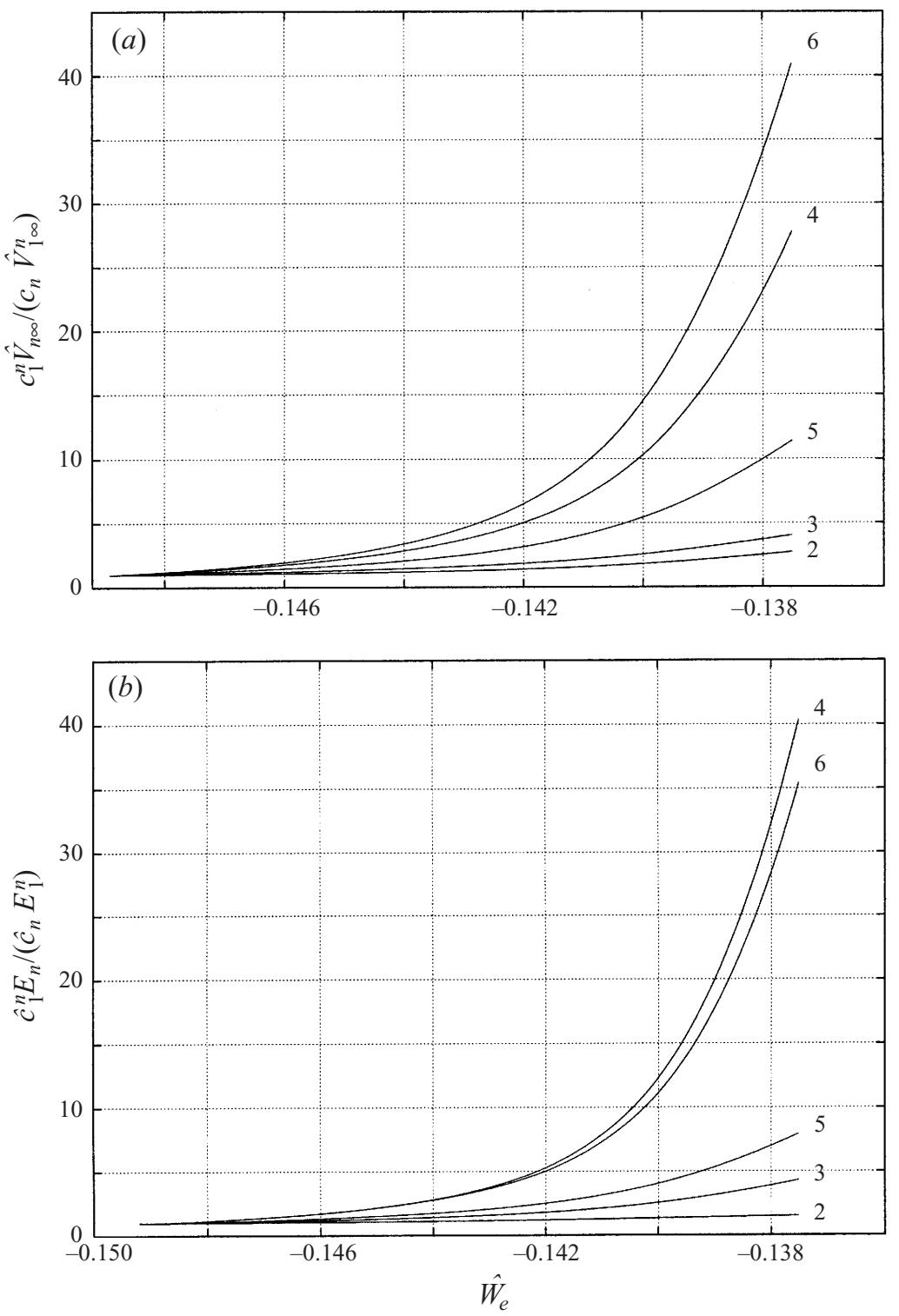

FIGURE 8 . The $n=1$ nonlinear travelling-wave solution for a flat-disk geometry: $(a)$ and $(b)$ show measures of the modes presented in figure 7. A deviation from unity shows the departure from a weakly nonlinear behaviour.

changes in $\hat{W}_{e}$ as the limit of our computations is approached. The breakdown of the numerical approach may also be connected to another suggested feature, namely $\hat{V}_{0 \infty} \rightarrow 0$. This condition is obviously significant in the exact solution of Appendix A, with $\hat{V}_{0 \infty}>0$ leading to (spatially) exponentially growing states. Nevertheless, without a corresponding asymptotic description of the more general states that displays these features, the failure of (3.1) remains a conjecture. However, the characteristic features noted for the failure of this $n=1$ solution appear to be generic, as we shall illustrate further by a nonlinear continuation of the $n=3$ state. 

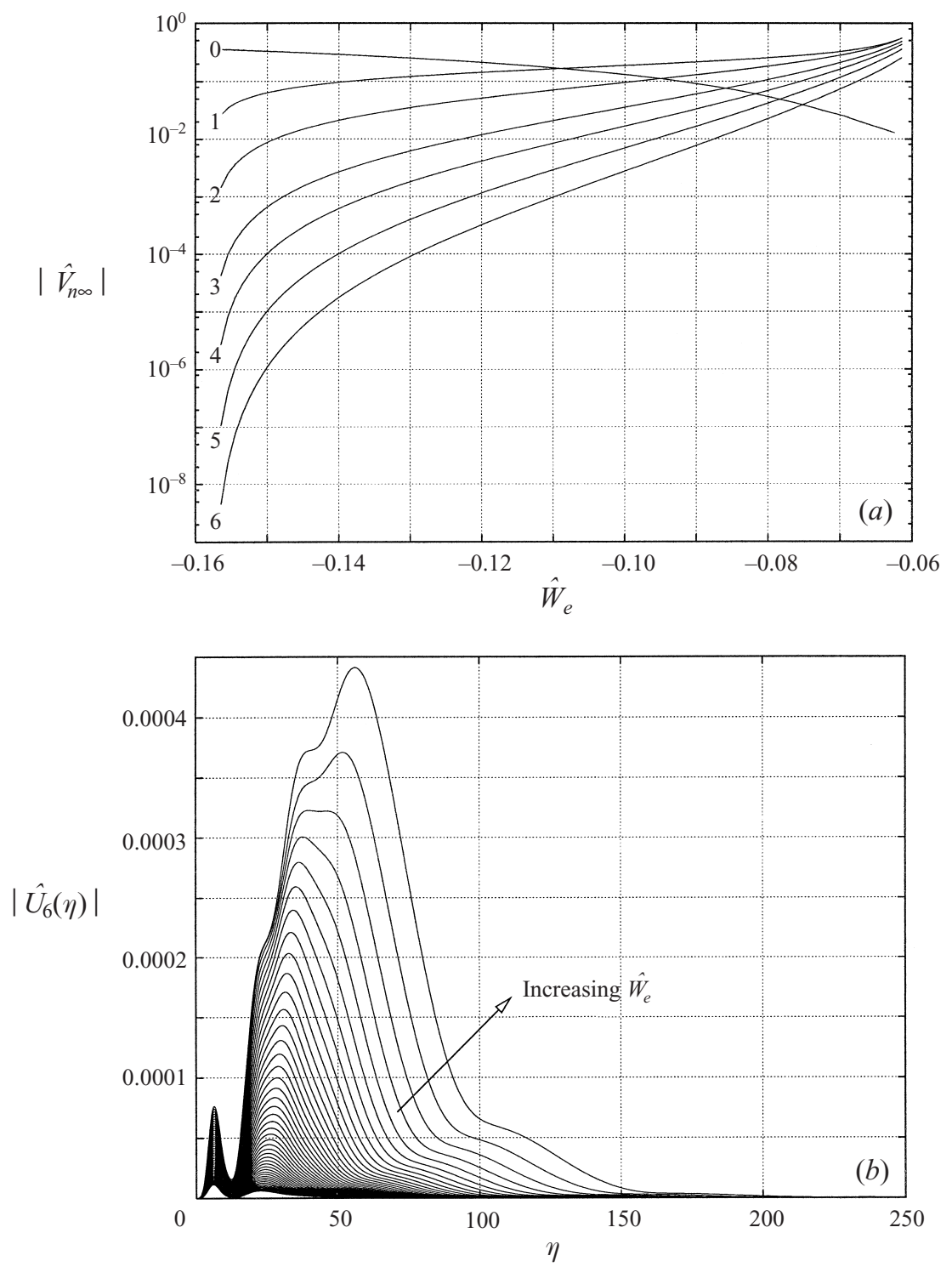

FiguRE 9. (a) The axial flow at infinity for the first six modes as the $n=3$ state is continued to finite amplitude. (b) Profiles of $\left|\hat{U}_{6}(\eta)\right|$ near the critical value of $\hat{W}_{e}$ at which the $n=3$ solution fails.

3.2. The $n=3$ state arising from a branch-2 axisymmetric state

As in the case of the $n=1$ state, the nonlinear continuation of the $n=3$ solution fails at a critical value of $\hat{W}_{e}$ with $\hat{V}_{0 \infty} \approx 0$. In this instance the failure of the series expansion (3.1) is more convincing, as can be seen by a comparison of any amplitude measure of the individual modes near the critical point. In figure $9(a)$ we take the axial flow at infinity as representing the amplitude of the modes, and compare them over the range of $\hat{W}_{e}$ for which numerical data can be obtained. It is easily seen that near the critical value the higher harmonics are all becoming of comparable size.

A typical velocity profile for varying $\hat{W}_{e}$ is shown in figure $9(b)$, clearly illustrating 


\begin{tabular}{ccc}
\hline & $A$ & $\Omega$ \\
3 & 0.5615 & 0.8361 \\
4 & 0.5825 & 0.8325 \\
5 & 0.5941 & 0.8309 \\
6 & 0.5992 & 0.8304 \\
8 & 0.6025 & 0.8302
\end{tabular}

TABLE 1. Variation of $A=\hat{U}_{1}^{\prime}(0)$ and $\Omega$ on the number of modes in the series (3.1) for the nonlinear state at $1 / \hat{W}_{e}=0$, computed with $10^{3}$ grid points in the domain $\eta \in[0,200]$.

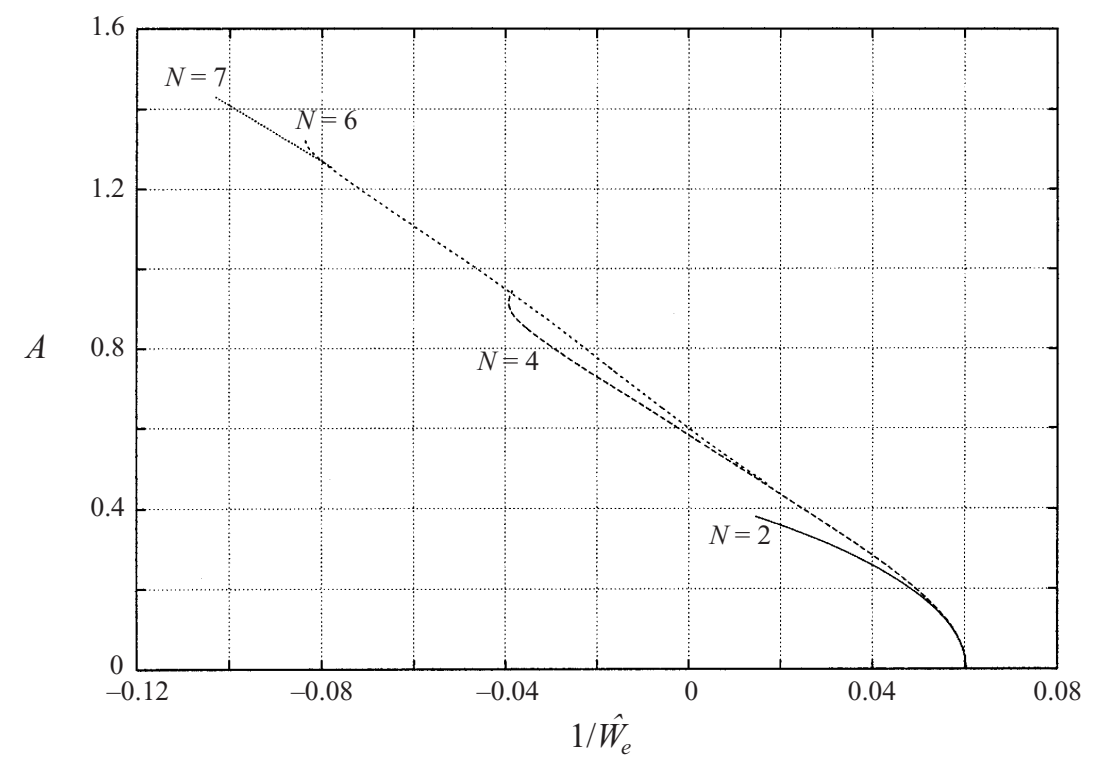

FiguRE 10. Variation of the amplitude measure, $A$, following the nonlinear state arising from the $n=1$ bifurcation near the Bödewadt limit. The effect of truncating the series (3.1) at $N=2,4,6,7$ is shown. There is a new, non-axisymmetric state predicted for $1 / \hat{W}_{e}=0, A \approx 0.6$; this is a boundary-layer alternative to the classical Bödewadt solution for a rotating fluid above a fixed plane.

a growing lengthscale as $\hat{W}_{e}$ approaches the limiting value. One may expect that a description of the large- $\eta$ behaviour may be obtainable; however, numerical results indicate that the velocity components for each mode decay (spatially) at a comparable rate. As a consequence, the $\hat{V} \hat{U}_{\eta}$ term in the governing equations ensures that all the modes remain coupled in any large- $\eta$ expansion.

\subsection{A nonlinear, non-axisymmetric alternative to the Bödewadt solution}

As we have noted in $\S 2.3$, there is a bifurcation to a non-axisymmetric travelling-wave state near $1 / \hat{W}_{e} \approx 0.06$, with $\Omega \approx 0.87$. By employing our numerical approach it is straightforward to compute a nonlinear continuation of this state.

The computations we present herein have been performed with a range of modes from 2 to 8 and a range of domains/grid sizes to ensure accurate computations. A truncation of the series (3.1) at $N=8$, with $10^{3}$ grid points over a domain of $\eta \in[0,200]$ was found to be sufficient to accurately determine the nonlinear, non- 

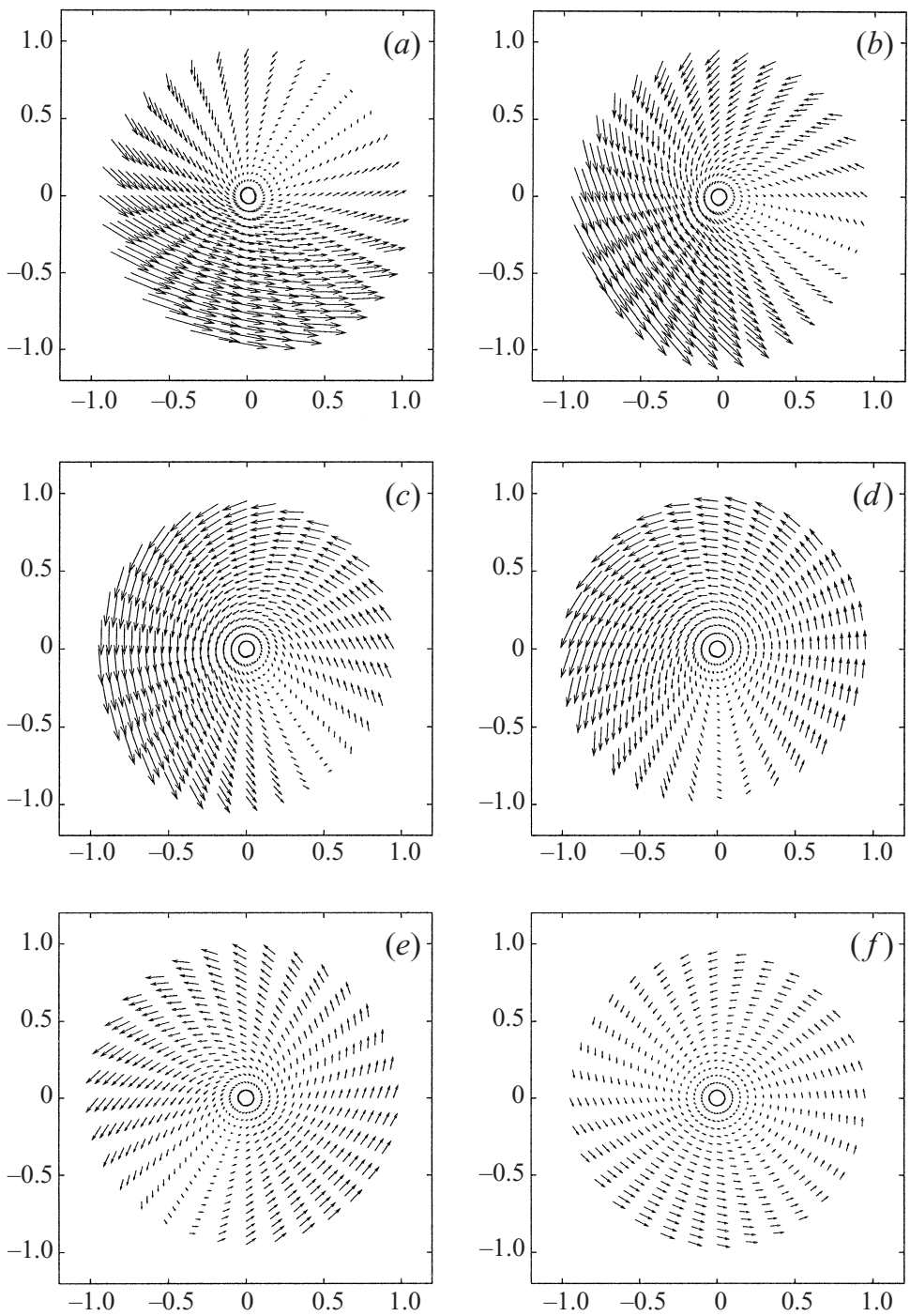

FIGURE 11. Instantaneous velocity fields in the $(r, \phi)$-plane at fixed values of $\eta$ for the nonlinear, non-axisymmetric, travelling-wave alternative to the Bödewadt solution (at $\left.1 / \hat{W}_{e}=0\right)$. The corresponding values of $\eta$ are $(a) \eta=2.5,(b) \eta=10,(c) \eta=17.5,(d) \eta=25$, (e) $\eta=32.5$, (f) $\eta=47.5$. All the velocity fields are presented with $\Omega t+\Delta=0$. This state has been computed with $N=8$ modes, and 1000 equally spaced grid points over the domain $\eta \in[0,200]$.

axisymmetric alternative to the Bödewadt solution at $1 / \hat{W}_{e}=0$. These values were a practical maximum for workstation-based computations of the solution branches due to the storage requirements of the solution method. We provide some numerical data on the effect of varying $N$ in table 1 .

Although this non-axisymmetric state can be continued to higher amplitude, as shown in figure 10, we note that in general additional modes must be included into the numerical scheme. Figure 10 clearly shows the bifurcation point and the presence of a nonlinear, non-axisymmetric state available when $1 / \hat{W}_{e}=0$. In figure 11 we illustrate the velocity field over a unit radial distance in the $(r, \phi)$-plane at fixed values 


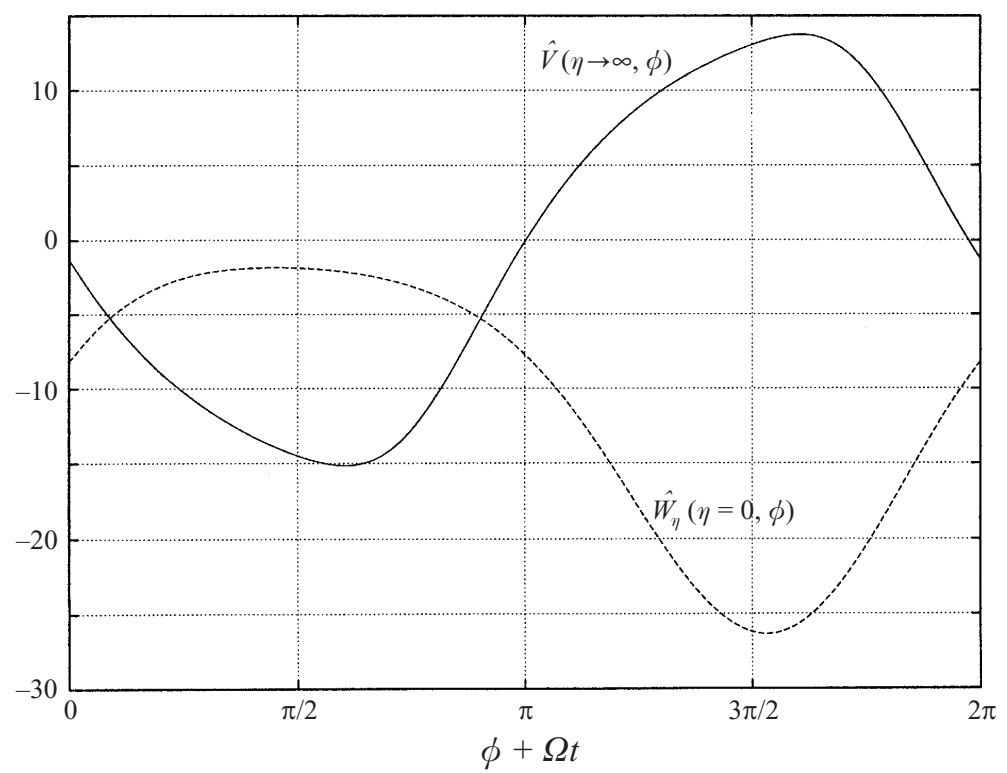

FIgURE 12 . The axial velocity at the edge of the boundary layer $\hat{V}(\eta \rightarrow \infty, \phi)$ and a shear stress component at the disk $\hat{W}_{\eta}(\eta=0, \phi)$ as a functions of $\phi$ at fixed time. These results are for the alternative Bödewadt state $\left(1 / \hat{W}_{e}=0\right)$ shown in figure 11 .

of $\eta$ for this new solution. Since $1 / \hat{W}_{e}=0$ there is no flow at $\eta=0$ since the disk is stationary.

In figure 12 we again refer to the same nonlinear state illustrated in figure 11; however in this case we show the mean axial velocity at infinity and a shear stress component at the disk over one period of the motion.

Finally, in figure 13 we show the velocity field at a fixed unit radius in the $(\eta, \phi+\Omega t)$ plane. As noted elsewhere (figure 12) the oscillatory axial flow at infinity is clearly seen.

\subsection{Temporally periodic axisymmetric states}

The temporal stability of the axisymmetric rotating-disk equations to axisymmetric modes has been considered by Bodonyi \& $\mathrm{Ng}(1984)$. These results showed a stability transition at the limit point of the solution branches shown in figure 1, with all higher-branch modes having at least one unstable eigenvalue. We must also note that the stability analysis is complicated by the presence of a damped continuous spectrum and some subtleties can arise that we shall not discuss here.

A further result of Bodonyi \& $\mathrm{Ng}$ is that, at a value of $1 / \hat{W}_{e} \approx-0.03$, a Hopf bifurcation leads to a limit-cycle solution to the axisymmetric rotating-disk equations. This result agrees with the data obtained for the initial-value problem as presented by Bodonyi (1978).

We do not give a detailed account of these states here, but we do note that the numerical method employed to calculate the nonlinear, non-axisymmetric states presented in the previous sections can also be applied to these axisymmetric, periodic states simply by taking $\lambda=0$. Apart from some minor quantitative differences arising from the limited resolution available to the initial-value calculations of Bodonyi (1978), we have been able to verify our current method by direct computation of the limit-cycle states. 


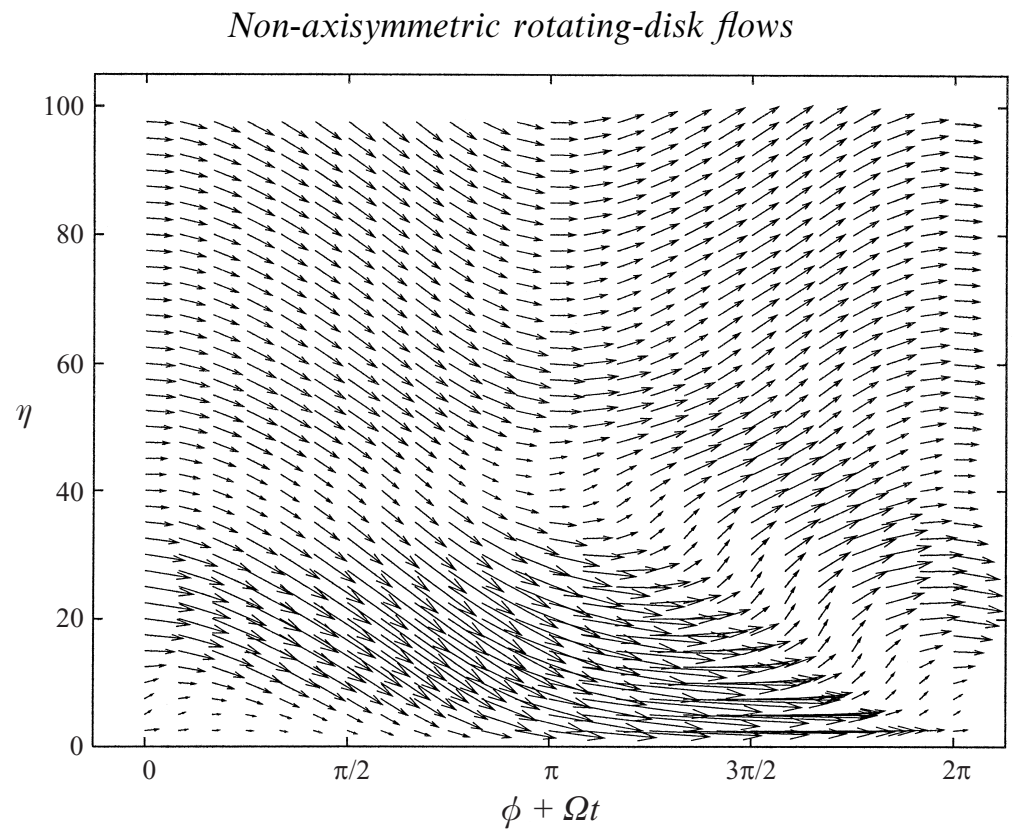

FIGURE 13 . The instantaneous velocity field at a fixed radius, in the $(\eta, \phi)$-plane for the alternative Bödewadt state (at $1 / \hat{W}_{e}=0$ ). As noted in the preceding figure, the axial flow at the edge of the boundary layer changes sign as $\phi$ varies. See figure 11 for details of the numerical parameters.

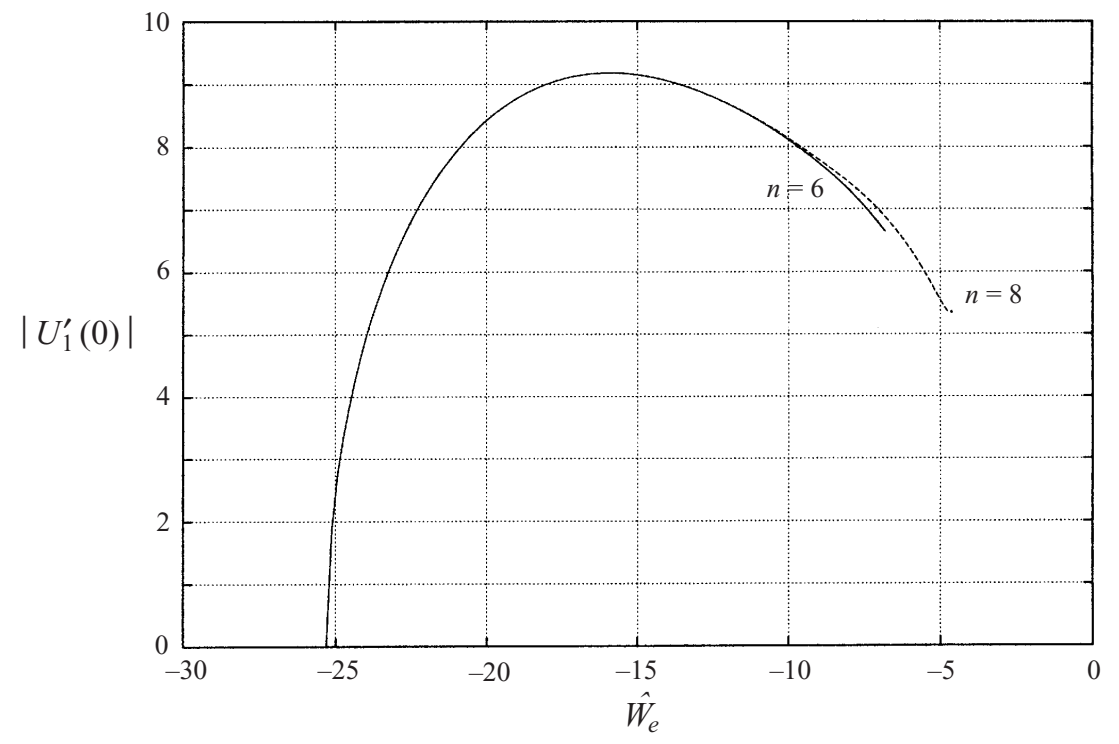

FIGURE 14. Nonlinear, temporally periodic, axisymmetric states arising from a Hopf bifurcation near $\hat{W}_{e} \approx-25.3$.

In figure 14 we present some details of the finite-amplitude limit-cycle solutions that arise from the Hopf bifurcation. The calculations shown in the figure have been performed with 451 equally spaced grid points over a domain of $\eta \in[0,30]$. It must be noted that further grid refinement can alter the location of the Hopf bifurcation slightly, providing a value of $1 / \hat{W}_{e} \approx-0.0367$ (compared to Bodonyi \& Ng's value of -0.03 ) but making little qualitative difference to the finite-amplitude states. 
As can be observed from figure 14, the computations with six modes terminated at a critical value of $\hat{W}_{e}$, but could be continued to slightly larger values (before terminating again) by increasing the number of harmonics to eight. It seems likely that the series representation fails at some critical value of $\hat{W}_{e}$ as has already been suggested for the non-axisymmetric states.

The structure of the limit-cycle solution branch is more complicated than shown in figure 14. We have computed the evolution for the initial-value problem corresponding to an initial state of rigid-body rotation at unit frequency, with an impulsive change in the rotation rate of the disk to $1 / \hat{W}_{e}$. Computations of this sort again confirm the finite-amplitude limit-cycle states described above, and have been presented previously by Bodonyi (1978). However, further investigation suggests that the limit-cycle state undergoes a period doubling at a critical value of $\hat{W}_{e}$. One may expect therefore that a Floquet analysis of the states shown in figure 14 will reveal a stability transition at a finite amplitude and the existence of a further (period doubled) branch appearing at some critical value of the rotation rate.

The initial-value problem for the flow over a rotating disk has also been considered by Bodonyi \& Stewartson (1977) and Stewartson, Simpson \& Bodonyi (1982), in which the boundary-layer structure was shown to break down at a finite time when $\hat{W}_{e}=-1$. Similar finite-time singularities can be located for $W_{\text {crit }}<\hat{W}_{e}<-0.1605$, that is, for a range of values beyond the limit point of the axisymmetric solution branches shown in figure $1(a)$. Outside this range, the boundary layer evolves to a branch-1 steady-state solution if $\hat{W}_{e}>-0.1605$ or, when in the range $W_{\text {Hopf }}<\hat{W}_{e}<W_{\text {crit }}$, a limit-cycle solution is achieved.

The boundary $\hat{W}_{e}=W_{\text {crit }}$, which separates an evolution to a limit cycle from one to a finite-time singularity, is not clearly defined in the existing literature. In fact, great care is required when performing computations relevant to the initial value problem in this region of parameter space. If multiple evolutions are obtained over a range of values of $\hat{W}_{e}$ (near $\hat{W}_{e}=W_{\text {crit }}$ ) and the post-transient behaviour is examined, it is possible to obtain a limit-cycle solution, followed by a period-doubling cascade to chaos and eventually a finite-time breakdown as $\hat{W}_{e}$ is increased. Nevertheless, it is far from clear that such chaotic behaviour is a genuine property of the continuous system rather than just the discretized equations. In particular, the region of parameter space in which chaotic behaviour is found is observed to shrink with continued grid refinement.

One possibility is that $W_{\text {crit }}$ may be associated with a point at which the finiteamplitude periodic solutions of figure 14 terminate with a singular structure, and beyond this point the temporal evolution of the solution ends in a finite-time breakdown.

\section{Discussion}

In this paper we have examined the flow above a rotating disk in a rotating fluid. By relaxing assumptions concerning the axisymmetry of the flow we have shown that a large class of alternative solutions is available to this classical configuration.

These alternative states are non-axisymmetric flow regimes, although the axisymmetric boundary conditions are still satisfied at the disk surface and at infinity. With the exception of one sub-class of flow (the $n=2$ mode), these states require a boundary-layer approximation based on a small Ekman number. The more general class of non-axisymmetric boundary-layer states takes the form of nonlinear waves that propagate azimuthally about the axis of rotation (in the sense of the disk/cone if 
$\Omega<0$, and in the opposite sense if $\Omega>0$ ). Although our nonlinear results concentrate on the flat-disk geometry, similar states relevant to a boundary layer near a conical wall can be developed by effectively taking a non-integer azimuthal wavenumber. Indeed, it was addressing the effect of a conical geometry on the exact $n=2$ (stationary) state that forced a consideration of travelling modes.

We have shown that there is an extremely broad range of non-axisymmetric states associated with the well-known non-uniqueness of the axisymmetric states. However, non-axisymmetric modes are not just found near to $\hat{W}_{e}=0$. By applying the same bifurcation analysis 'near' the classical axisymmetric Bödewadt solution, we have been able to generate a fully nonlinear, non-axisymmetric boundary-layer state as an alternative flow above a stationary plane in a rotating fluid.

Several of the solution branches have been continued from the bifurcation points to fully nonlinear non-axisymmetric states. In general, the states we have located either grow to amplitudes for which calculations are difficult (in terms of truncation of the series) or fail at finite values of $\hat{W}_{e}$ with what appears numerically to be some form of singular structure.

Showing the existence of non-axisymmetric states is of some interest in itself; however, for practical purposes a full and detailed consideration of the stability of these states is required. We do not provide such a description here, but we can make some comments on the temporal stability of the more easily located non-axisymmetric branches in the vicinity of the corresponding bifurcation point.

We must first preface any comments concerning the stability of such weakly nonlinear states by stating the class of perturbations to be considered. When discussing the stability of these states we are implicitly assuming the disturbance to be within the same class of solution, and therefore to possess the same radial similarity. We do recognise however that the stability of the states to other classes of perturbation can also be considered (for example, see Lingwood 1995). Indeed, it must also be noted that it is not always clear that a solution of von Kármán form is achievable even in a sub-region of a finite flow domain. Nevertheless, some comments on the stability to self-similar perturbations are important.

This work has essentially derived nonlinear travelling-wave states for a boundaryvalue problem, a procedure that has been applied to many other systems, and in particular arises when considering the stability of some plane channel flows. As in the case of channel flows, Stuart (1960), it is straightforward to generate a weakly nonlinear description of the wave solution in the vicinity of the bifurcation points. In particular, a perturbation expansion of the form

$$
\hat{W}_{e}=\hat{W}_{b i f}+\epsilon, \quad 0<\epsilon \ll 1,
$$

can be applied with

$$
\begin{aligned}
\boldsymbol{U}= & \boldsymbol{U}_{0}(\eta)+\epsilon^{1 / 2}\left\{A(\tau) \boldsymbol{U}_{11}(\eta) E+\text { c.c. }\right\} \\
& +\epsilon\left\{\boldsymbol{U}_{2}(\eta)+A_{20}(\tau) \boldsymbol{U}_{20}(\eta)+\boldsymbol{U}_{22}(\eta) E^{2}+\text { c.c. }\right\} \\
& +\epsilon^{3 / 2}\left\{A_{31}(\tau) \boldsymbol{U}_{31}(\eta) E+A_{33}(\tau) \boldsymbol{U}_{33}(\eta) E^{3}+\text { c.c. }\right\}+\cdots,
\end{aligned}
$$

where $\tau=\epsilon t, E=\exp [\mathrm{i}(\lambda \phi+\Omega t)]$, and $\lambda$ is required to be an integer for a flat-disk geometry.

In such an expansion, $\boldsymbol{U}_{11}(\eta)$ is normalized in some arbitrary manner and $A(\tau)$ is an amplitude measure. At $O\left(\epsilon^{0}\right)$ we obtain the rotating-disk equations, then at $O\left(\epsilon^{1 / 2}\right)$ the eigenvalue problem (2.2) is recovered, which determines the location of 
the bifurcation $\hat{W}_{\varepsilon}=\hat{W}_{b i f}$ and the real frequency of the wave, $\Omega$. At this stage the amplitude $A(\tau)$ is undetermined.

At $O(\epsilon)$, one determines $\boldsymbol{U}_{2}$ as a Taylor-series correction to the mean flow, while $A_{20}=A^{2}$ and $\boldsymbol{U}_{22}$ is derived from an inhomogeneous system. At $O\left(\epsilon^{3 / 2}\right)$ an orthogonality condition must be satisfied, which leads to the usual Stuart-Landau amplitude equation

$$
A_{\tau}=\sigma A-\beta A|A|^{2}
$$

with $\sigma$ and $\beta$ complex constants. The two constants can be obtained in the form of integrals of the lower-order solutions and the adjoint system. In fact, the complex constant $\sigma=\mathrm{i} \partial \Omega / \partial \hat{W}_{e}$ evaluated at $\hat{W}_{e}=W_{b i f}$, and can be obtained from a solution of the $O\left(\epsilon^{1 / 2}\right)$ eigenvalue problem by allowing for a complex $\Omega$.

This procedure can be applied to the range of solutions presented in this current work, including the axisymmetric limit-cycle behaviour described in $\S 3.4$. For the exact $n=2$ state described in Appendix A, the bifurcation is found to be subcritical, with nonlinear effects acting to destabilize the wave $\left(\sigma_{r}<0, \beta_{r}<0\right)$. One therefore expects the exact solution branch to be unstable unless re-stabilized at some finite amplitude. However, this suggests that in this region of parameter space, a threshold amplitude response should be found for perturbations (of this form) to the axisymmetric solution branches. This raises the question of the exact nature of the large-time evolution given such a perturbation, and in this way the stationary exact solution branch relates to the unsteady analysis of Hall et al. (1992).

For the non-axisymmetric states arising from higher-branch solutions, it must be noted that although a neutrally stable mode obviously exists at the bifurcation point, there are also other unstable modes of different azimuthal wavenumber. For example, at the bifurcation to the $n=1$ state described in $\S 3.1$ the axisymmetric state is also unstable to modes of azimuthal wavenumber 2. Similarly, near the bifurcation to the $n=3$ state, the axisymmetric solution is unstable to modes of azimuthal wavenumber $n=1,2$ and, in agreement with the results of Bodonyi \& $\mathrm{Ng}(1984), n=0$.

For the non-axisymmetric travelling-wave state arising near the Bödewadt limit the stability is somewhat different. The $n=1$ state near $1 / \hat{W}_{e} \approx 0.06$ arises through a supercritical bifurcation from the axisymmetric state $\left(\sigma_{r}>0, \beta_{r}>0\right.$ in an analogous expansion of the form $1 / \hat{W}_{e}=1 / W_{b i f}-\epsilon$, with $\left.\epsilon>0\right)$. Therefore, one expects the weakly nonlinear $n=1$ solution to be stable under our assumptions concerning the disturbance class. If this is the case, and the new branch does not destabilize beyond some fully nonlinear stage, it leaves open the possibility that the non-axisymmetric state of $\S 3.3$ is the appropriate radially self-similar solution for a rotating flow over an infinite fixed plane. Without a detailed linear stability analysis of the finiteamplitude travelling-wave states we cannot draw further conclusions about the new non-axisymmetric states and these details remain a subject for future work.

However, it must be noted that in the Bödewadt limit, the net mass transport in the layer above the disk is directed radially towards the axis of rotation. As a consequence one might expect edge effects to propagate inwards, increasing the likelihood of destroying any self-similarity in an experimental configuration. (For the fully non-axisymmetric state, the net transport of fluid is still directed inwards.) Little experimental work has been performed for this flow; however, the lower surface of a circular cylinder undergoing spin-down to rest can develop a Bödewadt-like flow at a sufficient distance from the sidewalls. In this geometry a critical Reynolds number of 25 (based on radial distance from the axis of rotation and initial rotation rate) has been reported by Savas (1987). Beyond this 'critical' radius, circular and spiral waves 
have been observed. In the experiments of Savas (1987), large-time measurements are prohibited by the effects of a centrifugal instability that convects from the cylindrical walls.

Much of the motivation for this work arose from observations of non-axisymmetric flow regimes in spin-down experiments involving conical containers and a stably stratified fluid. It is hoped that a continuation of the results presented herein to the case of a density-stratified fluid may allow for some detailed comparisons with laboratory data. We note that in such density stratified flows, the edge conditions are less of an issue since boundary-layer transport can be inhibited by buoyancy effects.

The authors would like to acknowledge the financial support of the EPSRC.

\section{Appendix A. Exact, non-axisymmetric, stationary states}

As has been noted by HDF, an exact, stationary, non-axisymmetric solution exists; in this appendix we present further properties of the solution. It is easy to verify that a steady solution to (1.3)-(1.6) exists in the form

$$
\begin{gathered}
\hat{U}=U_{0}(\eta)+U_{1}(\eta) \cos (2 \phi), \\
\hat{V}=V_{0}(\eta), \\
\hat{W}=W_{0}(\eta)-U_{1}(\eta) \sin (2 \phi), \\
Q_{\phi}=0,
\end{gathered}
$$

which also leads to an exact cancellation of the terms proportional to $E$ in the system (1.3)-(1.6). An azimuthal wavenumber of 2 is the only case in which such a cancellation occurs, whilst the $\pm U_{1}(\eta)$ coefficient of the $\phi$-dependence in the radial and azimuthal velocity components is required to satisfy continuity, since $\hat{V}$ remains axisymmetric.

This remarkable form of exact solution corresponds to a non-axisymmetric flow above a rotating disk; nevertheless, the axial velocity induced by mass transport in the boundary layer remains axisymmetric.

The solution (A 1)-(A 4) leads to a nonlinear governing system of the form

$$
\begin{gathered}
U_{0}^{2}+V_{0} U_{0}^{\prime}-W_{0}^{2}+U_{1}^{2}=U_{0}^{\prime \prime}-\hat{W}_{e}^{2}, \\
2 U_{0} W_{0}+V_{0} W_{0}^{\prime}=W_{0}^{\prime \prime}, \\
2 U_{0}+V_{0}^{\prime}=0, \\
2 U_{0} U_{1}+V_{0} U_{1}^{\prime}=U_{1}^{\prime \prime},
\end{gathered}
$$

to be solved subject to the boundary conditions

$$
U_{0}=V_{0}=U_{1}=0, \quad W_{0}=1 \quad \text { on } \quad \eta=0,
$$

and

$$
U_{0}, U_{1} \rightarrow 0, \quad W_{0} \rightarrow \hat{W}_{e} \quad \text { as } \quad \eta \rightarrow \infty .
$$

The large- $\eta$ structure of (A 5)-(A 10) is simply that of the rotating-disk equations, with the non-axisymmetric component in the form

$$
U_{1}(\eta) \sim u_{1} \exp \left\{\hat{V}_{\infty} \eta\right\} \quad \text { as } \quad \eta \rightarrow \infty .
$$

Therefore, we may only expect this class of solution to exist with $\hat{V}_{\infty}<0$. 


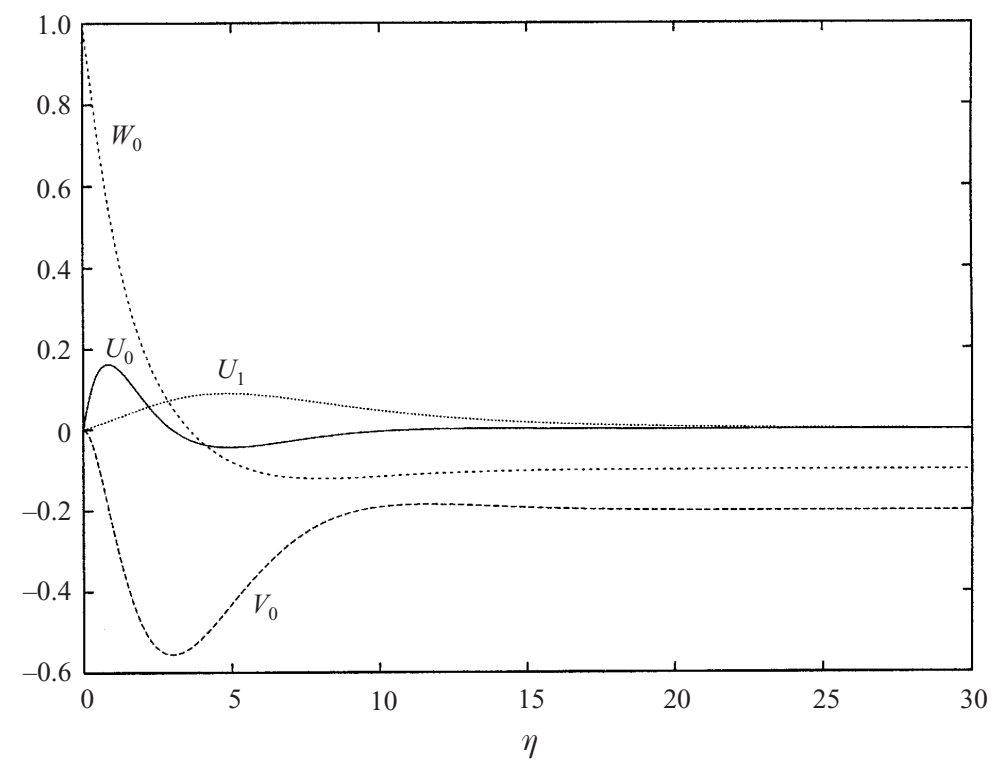

FIGURE 15 . Profiles of the components $U_{0}, V_{0}, W_{0}, U_{1}$ for a fully nonlinear non-axisymmetric state at $\hat{W}_{e}=-0.1, U_{1}^{\prime}(0) \approx 0.025$.

The system (A 5)-(A 10) still obviously admits solutions with $U_{1} \equiv 0$, that is, the classical axisymmetric rotating-disk states. However, as shown by HDF, there is a critical value of the parameter $\hat{W}_{e} \approx-0.14485$ at which a symmetry-breaking bifurcation occurs with a nonlinear non-axisymmetric solution available in the region $\hat{W}_{e} \in[-0.14485,0)$.

A figure showing the relative position of the bifurcated branch of non-axisymmetric solutions is given by HDF as figure 18 . We note that the behaviour of the nonaxisymmetric solutions as $\hat{W}_{e} \rightarrow 0^{-}$is non-trivial. The structure of the solution in this limit has been detailed by HDF; in particular, it has been shown that the lengthscale $\eta \sim\left|\hat{W}_{e}\right|^{-1 / 2}$ as $\hat{W}_{e} \rightarrow 0^{-}$.

Figure 15 shows profiles of the velocity components $U_{0}, V_{0}, W_{0}$ and $U_{1}$ for a fully nonlinear non-axisymmetric state at $\hat{W}_{e} \approx-0.1, V_{\infty} \approx-0.2, U_{1}^{\prime}(0) \approx 0.025$. In figure 16 , we show the velocity field

$$
U \hat{\boldsymbol{r}}+W \hat{\boldsymbol{\phi}}=r\left(U_{0}(\eta)+U_{1}(\eta) \cos 2 \phi\right) \hat{\boldsymbol{r}}+r\left(W_{0}(\eta)-U_{1}(\eta) \sin 2 \phi\right) \hat{\boldsymbol{\phi}},
$$

at fixed values of the scaled coordinate $\eta$, over a unit radial distance for the same nonlinear state presented in figure 15. As noted by Hall et al. (1992), the form of the non-axisymmetry in this class of solution when transformed into Cartesian coordinates is a stagnation-point flow. This can be readily seen in figure $16(c)$, since at this value of $\eta(\approx 3.6), U_{0}$ and $W_{0}$ are sufficiently small for the velocity field to be dominated by the $U_{1}$ non-axisymmetric components.

As shown in figure 16, when sufficiently close to (or far from) the disk surface the flow is in rigid-body rotation. Since $\hat{W}_{e}<0$ the far-field fluid and the disk counterrotate, as can be seen by comparing $16(a)$ and $16(f)$. Similarly, since $\hat{V}_{\infty}<0$, the axial flow at 'infinity' is towards the disk, and the net transport near to the disk is radially outwards. 

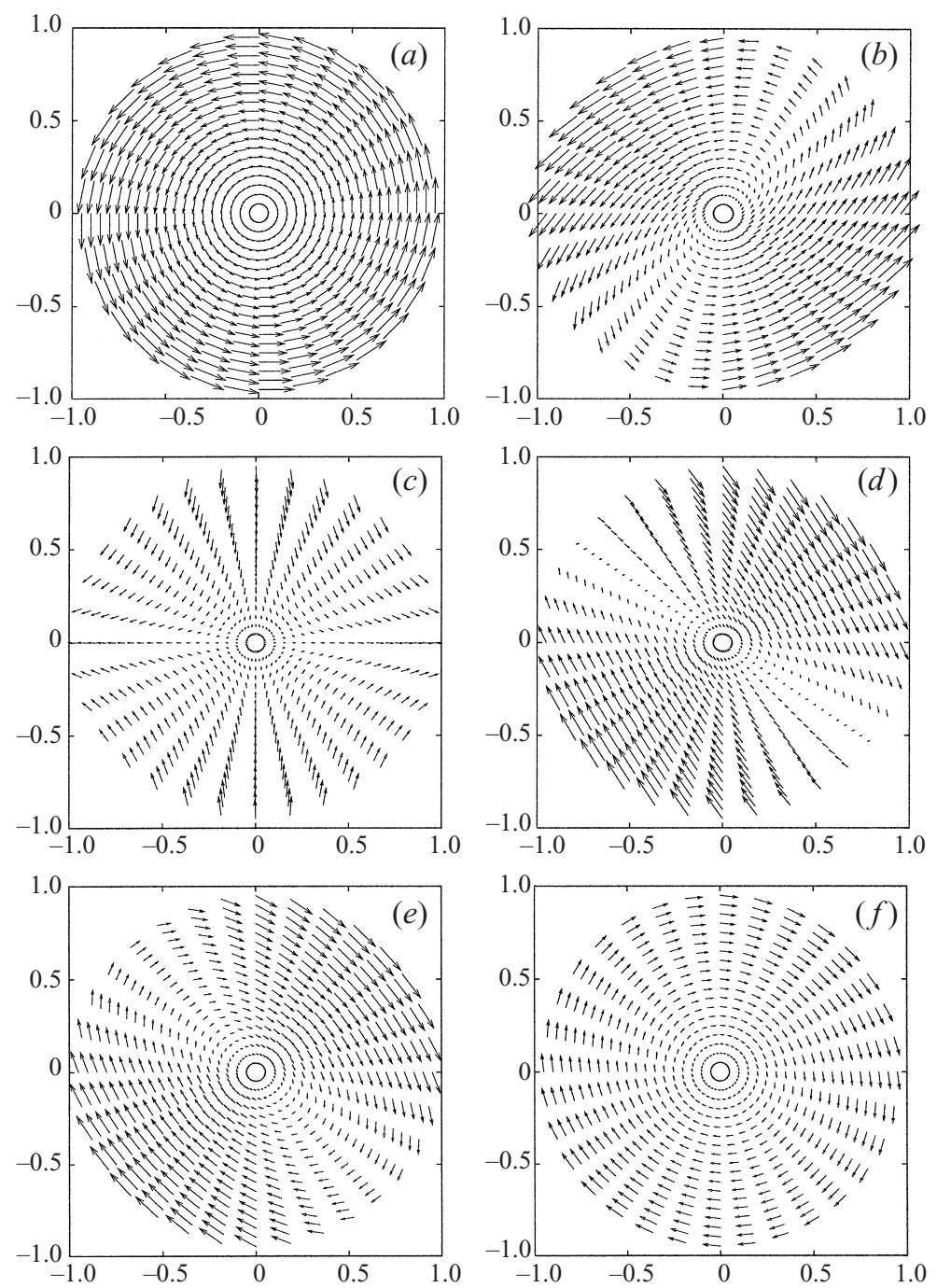

FIGURE 16. Velocity fields at fixed values of $\eta$ for a nonlinear, non-axisymmetric state at $\hat{W}_{e} \approx-0.1$, $\hat{V}_{\infty} \approx-0.2, U_{1}^{\prime}(0) \approx 0.025$. The corresponding values of $\eta$ are $(a) \eta=0,(b) \eta \approx 2.41,(c) \eta \approx 3.62$, (d) $\eta \approx 5.43,(e) \eta \approx 9.05,(f) \eta \approx 16.88$. (The velocity field in $(a)$ is scaled by a factor of 5 ).

\section{Appendix B. An extension to a conical geometry}

Here we provide some details on how to extend the analysis presented in Appendix A to a conical geometry. Although, at first sight, this may seem superfluous, it is the non-trivial nature of this simple extension that forces the consideration of other travelling-wave states, showing that the exact solution is simply one of a broader class of solutions.

It has been shown by HDF, and by Duck, Foster \& Hewitt (1997), that the equations governing the axisymmetric boundary layer on the inside of a rotating cone in a rotating homogeneous fluid can also be reduced to the rotating-disk equations (1.9)-(1.11). As noted by HDF however, when non-axisymmetric flows are considered 
the governing equations reduce to

$$
\begin{gathered}
\hat{U}^{2}+\hat{V} \hat{U}_{\eta}-\hat{W}^{2}+\beta^{-1} \hat{W} \hat{U}_{\phi}=\hat{U}_{\eta \eta}-\hat{W}_{e}^{2} \\
2 \hat{U} \hat{W}+\hat{V} \hat{W}_{\eta}+\beta^{-1} \hat{W} \hat{W}_{\phi}=\hat{W}_{\eta \eta}, \\
\hat{V} \hat{V}_{\eta}+\beta^{-1} \hat{W} \hat{V}_{\phi}=-Q_{\eta}+\hat{V}_{\eta \eta}, \\
2 \hat{U}+\hat{V}_{\eta}+\beta^{-1} \hat{W}_{\phi}=0 .
\end{gathered}
$$

The conical shell is orientated with the walls at an angle of $\pi / 2-\alpha$ to the axis of rotation (as shown in figure 2), and the parameter $\beta$ has been introduced, where

$$
\beta=\cos \alpha \text {. }
$$

Note that as $\alpha \rightarrow 0$ (a flat disk), $\beta \rightarrow 1$ and (B 1)-(B 4) reduces to the nonaxisymmetric version of the rotating-disk equations (1.3)-(1.6) under a boundarylayer approximation. This system is formally obtained following a boundary-layer approximation based on $E \ll 1$ applied to the full governing system in a sphericalpolar coordinate system centred at the apex of the cone.

Although the appropriate coordinate system is spherical in this case, the same notation applied to the flow over a disk has been re-used here for simplicity; for example, in this case the boundary-layer coordinate is defined $E^{1 / 2} \eta=(\pi / 2-\alpha)-\theta$, where $\theta$ is the meridional coordinate measured from the axis of rotation. Here $\hat{U}, \hat{V}$ denote velocity components that are parallel to the boundary in the radial direction and normal to the boundary respectively, while $\hat{W}$ is the azimuthal component.

The boundary conditions for (B 1)-(B 4) are as for the flat-disk case, with an axisymmetric rigid-body rotation (about the axis of symmetry of the cone) far from the boundary, together with no-slip and impermeability conditions on the wall.

It is the minor difference between (B 1)-(B 4) and the exact system (1.3)-(1.6) that allows some interesting comments to be made concerning the form of non-axisymmetric states in the conical geometry, which in turn leads to a further extension of the class of non-axisymmetric solutions. It can be seen immediately that a transformation

$$
\frac{\partial}{\partial \phi} \rightarrow \frac{1}{\beta} \frac{\partial}{\partial \phi}
$$

in the non-axisymmetric form of the rotating-disk equations (1.3)-(1.6) is sufficient to consider the equivalent conical analogue (B 1)-(B 4). Since the conical description can be obtained through this transformation, we can apply the previous results concerning the location of the bifurcation point to the conical geometry with no further work.

The bifurcation to an exact non-axisymmetric solution (as discussed in Appendix A) is obtained by effectively looking for weakly non-axisymmetric solutions in the form

$$
(\hat{U}, \hat{W}, \hat{V})^{T}=\left(U_{0}(\eta), W_{0}(\eta), V_{0}(\eta)\right)^{T}+\epsilon(\tilde{U}(\eta), \tilde{W}(\eta), \tilde{V}(\eta))^{T} \exp \{\operatorname{in} \phi\},
$$

where $\epsilon \ll 1$. In this way, $\left(U_{0}, W_{0}, V_{0}\right)$ are required to satisfy the axisymmetric rotating-disk equations, while the $O(\epsilon)$ terms provide a linear eigenvalue problem

$$
\mathscr{F}\left(\hat{W}_{e}, n\right)=0 \text {. }
$$

This eigenvalue relation determines a set of complex eigenvalues $n_{i}$ for a given real value of $\hat{W}_{e}$ (and associated leading-order rotating-disk solution). The location of the bifurcation point is then obtained as the value of $\hat{W}_{e}$ for which a real eigenvalue 
exists that is also an integer (to satisfy the azimuthal periodicity constraint). In this way we have shown that an azimuthally periodic state can be found with

$$
n=2 \text { at } \hat{W}_{e} \approx-0.14485 \text {. }
$$

Given the transformation of flat-disk solutions to conical solutions through (B 6), we can immediately observe that when $\alpha \neq 0$ the equivalent eigenvalue problem is obtained in the form

$$
\mathscr{F}\left(\hat{W}_{e}, n \beta^{-1}\right)=0 .
$$

It is therefore trivial to see that the linear eigenvalue relation has a solution with

$$
\frac{n}{\cos \alpha}=2 \text { at } \hat{W}_{e} \approx-0.14485 \text {. }
$$

However, since the bifurcated solutions are required to be periodic in the azimuthal direction, it is necessary for $n$ to be an integer (the azimuthal wavenumber). We are therefore forced to conclude that a bifurcation can only be found to a nonaxisymmetric state with azimuthal wavenumber $n=2$ when $\alpha=0$ (the flat disk) or a wavenumber of $n=1$ when $\alpha=\pi / 3$ (a conical container, with $\cos \alpha=1 / 2$ ).

The description of this $n=2$ mode, as presented in Appendix $\mathrm{A}$, is therefore incomplete in the sense that it fails to provide any non-axisymmetric states for any cone angles other than $\alpha=0$ or $\alpha=\pi / 3$. It seems counter-intuitive to suppose that the non-axisymmetric branch of solutions cannot be continued in the parameter $\alpha$, since the geometry can be made conical in a continuous manner starting from a flat disk solution.

To resolve how the $n=2$ bifurcation for the flat disk $(\alpha=0)$ and the $n=1$ bifurcation in the conical case $(\alpha=\pi / 3)$ are connected, one must seek a wider class of solution in a form described in $\S 2.1$.

\section{REFERENCES}

BAtChELOR, G. K. 1951 Note on a class of solutions of the Navier-Stokes equations representing steady rotationally-symmetric flow. Q. J. Mech. Appl. Maths 4, 29.

BöDEwadt, U. T. 1940 Die Drehströmung über festem Grund. Z. Angew. Math. Mech. 20, 241.

BodonyI, R. J. 1978 On the similarity equations for the flow above a rotating disc in a rotating fluid. Q. Appl. Maths Mech 31, 461.

BodonYI, R. J. \& NG, B. S. 1984 On the stability of the similarity solutions for swirling flow above an infinite rotating disk. $J$. Fluid Mech. 144, 311.

Bodonyi, R. J. \& STEWARTSON, K. 1977 The unsteady laminar boundary layer on a rotating disk in a counter-rotating fluid. J. Fluid Mech. 79, 669.

Duck, P. W., Foster, M. R. \& Hewitt, R. E. 1997 On the boundary layer arising in the spin-up of a stratified fluid in a container with sloping walls. J. Fluid Mech. 335, 233.

GreensPan, H. P. \& Howard, L. N. 1963 On a time-dependent motion of a rotating fluid. J. Fluid Mech. 17, 385 .

Hall, P., Balakumar, P. \& Papageorgiou, D. 1992 On a class of unsteady three-dimensional Navier-Stokes solutions relevant to rotating disc flows: threshold amplitudes and finite-time singularities. J. Fluid Mech. 238, 297.

Hewitt, R. E., Duck, P. W. \& Foster, M. R. 1999 Steady boundary-layer solutions for a swirling stratified fluid in a rotating cone. J. Fluid Mech. 384, 339 (referred to herein as HDF).

Kármán, T. von. 1921 Über laminare und turbulente Reibung. Z. Angew. Math. Phys. 1, 244.

Lai, C. Y., Rajagopal, K. R. \& Szeri, A. Z. 1985 Asymmetric flow above a rotating disk. J. Fluid Mech. 157, 471.

LingwoOd, R. J. 1995 Absolute instability of the boundary layer on a rotating disk. J. Fluid Mech. 299, 17.

SAvas, O. 1987 Stability of Bödewadt flow. J. Fluid Mech. 183, 77. 
Stewartson, K., Simpson, C. J. \& Bodonyi, R. J. 1982 The unsteady laminar boundary layer on a rotating disk in a counter-rotating fluid. Part 2. J. Fluid Mech. 121, 507.

StUART, J. T. 1960 On the nonlinear mechanics of wave disturbances in stable and unstable parallel flows. J. Fluid Mech. 9, 353 .

Zandbergen, P. J. \& Dijkstra, D. 1987 Von Kármán swirling flows. Ann. Rev. Fluid Mech. 19, 465. 